See discussions, stats, and author profiles for this publication at: https://www.researchgate.net/publication/319474824

\title{
Convergence regions for the Chebyshev-Halley family
}

Article in Communications in Nonlinear Science and Numerical Simulation · September 2017 DOI: 10.1016/j.cnsns.2017.08.024

CITATIONS

0

3 authors:

6.)

Beatriz Campos

Universitat Jaume I

37 PUBLICATIONS 69 CITATIONS

SEE PROFILE

P. Vindel

Universitat Jaume I

50 PUBLICATIONS 266 CITATIONS

SEE PROFILE
READS

77

Some of the authors of this publication are also working on these related projects:

Singular perturbations of Blaschke products. View project 


\title{
Convergence regions for the Chebyshev-Halley family
}

\author{
B. Campos, J. Canela and P. Vindel \\ campos@uji.es, canela@maia.ub.es, vindel@uji.es
}

\begin{abstract}
In this paper we study the dynamical behavior of the Chebyshev-Halley methods on the family of degree $n$ polynomials $z^{n}+c$. We prove that, despite increasing the degree, it is still possible to draw the parameter space by using the orbit of a single critical point. For the methods having $z=\infty$ as an attracting fixed point, we show how the basins of attraction of the roots become smaller as the value of $n$ grows. We also demonstrate that, although the convergence order of the Chebyshev-Halley family is 3, there is a member of order 4 for each value of $n$.

In the case of quadratic polynomials, we bound the set of parameters which correspond to iterative methods with stable behaviour other than the basins of attraction of the roots.
\end{abstract}

\section{Introduction}

Numerical methods are the main tool used by scientists and engineers for finding solutions of equations that can not be solved analytically. This encourages mathematicians, not only to seek new numerical methods, but also to study and improve the methods already known.

For the case of nonlinear equations, it is usual to consider iterative methods with high order of convergence to approximate the solutions. However, the radii of convergence which ensure that the solution of the method is correct are small. Accordingly, to improve the numerical methods and to expand their radii of convergence is one of the challenges to which mathematicians are faced. An overview of the analysis of different iterative methods can be found in [?]. In this book, besides studies dealing with the design, convergence, efficiency and robustness of the methods, we can also find an introduction to dynamical analysis of iterative methods.

The study of iterative methods from the point of view of dynamical systems gives a new perspective that allows to carry out a study of the qualitative behavior of such methods, establishing conditions of stability. It also enables us to study families of methods in terms of parameters, analyzing which members of the family work better.

The best known iterative method, under the dynamical point of view, is Newton's scheme (see [?]). In previous papers the dynamics of other iterative families are investigated: the Chebyshev-Halley family ([?], [?], [?]), the King's class [?], the $c$-family [?] and the $(\alpha, c)$ family [?], which includes Chebyshev-Halley and $c$ families. In these papers the dynamical behaviour of the iterative methods on quadratic polynomials is studied and regions with no convergence to the roots are found. Different authors have studied other point-to-point iterative methods for solving nonlinear equations (see [?], [?], [?], [?], [?],.. for example). 
For the case of the Chebyshev-Halley type methods, the corresponding fixed point operator is

$$
G(z)=z-\left(1+\frac{1}{2} \frac{L_{f}(z)}{1-\alpha L_{f}(z)}\right) \frac{f(z)}{f^{\prime}(z)}
$$

where

$$
L_{f}(z)=\frac{f(z) f^{\prime \prime}(z)}{\left(f^{\prime}(z)\right)^{2}}
$$

and $\alpha$ is a complex parameter. This family gives rise to widely known and used numerical methods for different values of the parameter, such as the Chebyshev's method for $\alpha=0$, Halley's scheme for $\alpha=\frac{1}{2}$, super-Halley's method for $\alpha=1$ and Newton's method when $\alpha$ tends to $\infty$.

The aim of this paper is twofold. Firstly, we continue the dynamical study of the ChebyshevHalley family applied on quadratic polynomials ([?]). For this case, we bound the set of parameters corresponding to iterative methods with stable behaviour other than the basins of attraction of the roots (Theorem 4.1). We also study the values of the parameters for which the strange fixed points are indifferent (Propositions 5.1 and 5.2). By doing this, we locate attracting cycles which come from bifurcations of rationally indifferent fixed points as well as Siegel disks.

Secondly, we study the Chebyshev-Halley methods using the family of degree $n$ polynomials $z^{n}+c$. The studies of numerical methods published to the date only consider low degree polynomials. We prove that, despite increasing the degree, it is still possible to determine the existence of attractors by using the orbit of a single critical point. In fact, in Section 6 we found $n$ free critical points, that is, critical points that do not match the roots. We prove that they are symmetric with respect to the $n t h$-roots of the unity and so are their orbits. Hence, it is enough to analyze the asymptotic behaviour of one of the $n$ critical orbits to comprehend the asymptotic behaviour for all of them (Lemma 6.2 ).

We want to remark that the parametrizations used in both studies are different. For the study of the Chebyshev-Halley family applied on quadratic polynomials, infinity is associated with one of the roots of the polynomial, so, it is always a superattracting fixed point. For the general study of the Chebyshev-Halley family applied on $n$-degree polynomials, although infinity is a fixed point, it is not always attracting (Proposition 6.1). Moreover, we observe that when infinity is attracting, the basins of attraction of the roots become smaller as the value of $n$ grows.

Taking into account these results we draw the parameter spaces of this family of maps. We show the parameter spaces for $n=2,3,4,5,10,100$. In all figures, we can see the black disk of parameters described in Proposition 6.1 for which the strange fixed point $z=\infty$ is attracting. We can also observe that the bound on the set of parameters with bad numerical behaviour which we obtain in the case of the quadratic polynomials $z^{2}+c$ also applies for the $z^{n}+c$ studied numerically.

We show the dynamical planes for $n=2,3,5$ and 10 for some values of the parameter $\alpha$ of the Chebyshev-Halley family. When the value of the parameter is $\alpha=\frac{n}{n-1}$, and hence $z=\infty$ is superattracting (Proposition 6.1), we observe that the basins of attraction of the roots become smaller when $n$ increases .

Finally, although the convergence order of the Chebyshev-Halley family is 3, we demonstrate that there is a member of order 4 for each value of $n$. (Proposition 6.3).

Let us recall some definitions used in complex dynamics. Given a rational map $R: \widehat{\mathbb{C}} \rightarrow \widehat{\mathbb{C}}$, where $\widehat{\mathbb{C}}$ denotes the Riemann sphere, the dynamical system given by its iterates is considered. 
The orbit $\mathcal{O}(w)$ of a point $w \in \widehat{\mathbb{C}}$ is given by the subsequent iterates of $w$ under $R(z)$, i.e.

$$
\mathcal{O}(w)=\left\{w, R(w), R^{2}(w), \ldots, R^{n}(w), \ldots\right\}
$$

A point $z_{0} \in \widehat{\mathbb{C}}$ is called fixed if $R\left(z_{0}\right)=z_{0}$. A point $z_{0}$ is called periodic of period $p>1$ if $R^{p}\left(z_{0}\right)=z_{0}$ and $R^{k}\left(z_{0}\right) \neq z_{0}, k<p$. A point $z_{0}$ is pre-periodic if it is not periodic but it is eventually mapped under iteration of $R(z)$ to a periodic one.

Fixed points can be classified depending on their multiplier $\lambda=R^{\prime}\left(z_{0}\right)$. A fixed point $z_{0}$ is called

- attractor if $|\lambda|<1$ and superattractor if $\lambda=0$;

- repulsor if $|\lambda|>1$;

- indifferent if $|\lambda|=1$.

An indifferent fixed point $z_{0}$ has multiplier $\lambda=R^{\prime}\left(z_{0}\right)=e^{2 \pi i \theta}$ with $\theta \in[0,1)$. Depending on this $\theta$, an indifferent fixed point is called

- rationally indifferent (or parabolic) if $\theta=p / q$, where $p, q \in \mathbb{N}$ are coprime numbers;

- irrationally indifferent if $\theta \in \mathbb{R} \backslash \mathbb{Q}$.

The same classification can be used for periodic points of any given period $p$ since they are fixed points of the map $R^{p}(z)$. The multiplier $\lambda$ of a fixed point $z_{0}$ determines the possible dynamics which might take place in a small neighbourhood of it (see [?]). Indeed, if $z_{0}$ is an attracting (or superattracting) fixed point, then there is an open neighbourhood $U$ of $z_{0}$ such that all points in $U$ accumulate under iteration of $R(z)$ in $z_{0}$, i.e. if $w \in U$ then $\lim _{n \rightarrow \infty} R^{n}(w)=$ $z_{0}$. Similarly, repelling fixed points repel the orbits of the points in a neighbourhood of them (they are attracting points of $R^{-1}(z)$ ).

The dynamics in a neighbourhood of indifferent points is more complicated than the one around attracting or repelling. It depends strongly on whether the point is rationally or irrationally indifferent. It follows from Leau-Fatou Flower theorem (see [?, Thm. 10.7]) that if a point $z_{0}$ is rationally indifferent then $z_{0}$ lies in the boundary of (at least) $q$ parabolic basins, open connected domains whose points tend under iteration to $z_{0}$. Moreover, these parabolic basins are mapped among themselves with period $q$ (see Figure 2 and Figure 3). On the other hand, if $z_{0}$ is an irrational fixed point then there might be an open neighbourhood $U$ of $z_{0}$ where the map $O_{\alpha}(z)$ is conjugate to the rigid rotation $R_{\theta}(z)=e^{2 \pi i \theta} z$. In that case, the maximal such domain $U$ is called Siegel disk (see Figure 4 and Figure 5).

The basin of attraction $\mathcal{A}\left(z_{0}\right)$ of an attracting point $z_{0}$ consists of the set of points $z \in \widehat{\mathbb{C}}$ that accumulate on $z_{0}$ under iteration of $R(z)$, i.e.

$$
\mathcal{A}\left(z_{0}\right)=\left\{z \in \widehat{\mathbb{C}}: R^{n}(z) \rightarrow z_{0} \text { when } n \rightarrow \infty\right\} .
$$

Similarly, the basin of attraction $\mathcal{A}\left(z_{0}\right)$ of a rationally indifferent point $z_{0}$ consists of the set of points $z \in \widehat{\mathbb{C}}$ that accumulate on $z_{0}$ under iteration of $R(z)$ but do not fall under iteration on $z_{0}$, i.e.

$$
\mathcal{A}\left(z_{0}\right)=\left\{z \in \widehat{\mathbb{C}}: R^{n}(z) \neq z_{0} \quad \forall n \in \mathbb{N}, R^{n}(z) \rightarrow z_{0} \text { when } n \rightarrow \infty\right\}
$$


The dynamics of $R(z)$ provides a totally invariant partition of the Riemann sphere. The Fatou set, $\mathcal{F}(R)$, of a rational map $R(z)$ consists of the points $z \in \widehat{\mathbb{C}}$ such that the family of iterates of $R(z),\left\{R(z), R^{2}(z), \ldots, R^{n}(z), \ldots\right\}$, is normal in some open neighbourhood $U$ of $z$. Its complement, the Julia set $\mathcal{J}(R)$, consists of the points where the dynamics of $R(z)$ is chaotic. The Fatou set is open and the Julia set is closed. The Julia set either has empty interior or coincides with $\widehat{\mathbb{C}}$. The connected components of the Fatou set are called Fatou components and are mapped among themselves under iteration. The celebrated result of D. Sullivan [?] states that all Fatou components of a rational map are either periodic or preperiodic. The Classification Theorem (see e.g. [?]) states that all periodic Fatou components are either basins of attraction of attracting or parabolic cycles, or simply connected rotation domains (Siegel disks) or doubly connected rotation domains (Herman rings).

The critical points of a rational map $R(z)$ are defined as the $z \in \widehat{\mathbb{C}}$ where $R(z)$ fails to be injective in any neighborhoud of $z$ or, equivalently, the $z \in \widehat{\mathbb{C}}$ such that $R^{\prime}(z)=0$ (see [?], for example). It is known that all periodic Fatou components are related to critical points (c.f. [?]). More specifically, the basins of attraction of attracting and rationally indifferent points contain, at least, a critical point while the orbit of, at least, a critical point accumulates on each connected component of the boundaries of Siegel disks and Herman rings.

The Fatou set for Newton's method on quadratic polynomials consists of the two basins of attraction of the superattracting fixed points, corresponding to the roots, while the Julia set consists of a straight line which separates these basins of attraction. However, as it can be seen in [?], the Julia set for Chebyshev-Halley's method applied on quadratic polynomials is more complicated than for Newton's method. A remarkable set, called Cat set, appears in the parameter space associated to the family. Previous results on this family are given in Section 2 .

The rest of the paper is organized as follows. In Section 3 we analyze the critical points and name them depending on their moduli in order to avoid problems with the choice of the determination of the square root. We also show that the orbits of the critical points are not independent. Afterwards, we use these critical points to plot the parameter space of the family.

In Section 4 we prove that the Cat set is bounded. This property guarantees that, if the value of the parameter is big enough, then the behaviour of the associated numerical method is suitable, that is, the orbit of almost any initial condition converges to one of the roots. Although a similar result can be seen in [?], they provide no estimation of how large the bound is, which is a drawback when analysing the validity of a numerical experiment. In Theorem 4.1 we prove that the numerical methods coming from this family are suitable for $|\alpha|>8.57$.

In Section 5 we study the boundary of the Cat set. We show how rationally indifferent fixed points and Siegel disks appear for parameters in the boundary of two given components.

In Section 6 we provide numerical studies of the behaviour of the Chebyshev-Halley methods on the family of degree $n$ polynomials $z^{n}+c$. First we analyse the stability of the fixed point $z=\infty$. We continue justifying that it is enough to analyse the orbit of one critical point to determine the existence of any stable behaviour other than the basins of attractions of the roots. Afterwards, we show that, fixed $n \geq 2$, there exists a unique parameter for which the method has order of convergence 4. Finally, we provide numerical examples of parameter spaces and dynamical planes for different values of the parameters. 


\section{Previous results on Chebyshev-Halley family}

The study of the dynamics of the operator (1) when it is applied on the quadratic polynomials $p(z)=z^{2}+c, c \in \mathbb{C}$, began in [?]. For these polynomials, the operator becomes the oneparametric rational function

$$
O_{\alpha}(z)=z^{3} \frac{z-2(\alpha-1)}{1-2(\alpha-1) z}
$$

after applying the conjugacy map $h(z)=\frac{z-i \sqrt{c}}{z+i \sqrt{c}}$.

The fixed points of this operator are $z=0, z=\infty, z=1$ and $s_{1,2}=(-3+2 \alpha \pm$ $\left.\sqrt{5-12 \alpha+4 \alpha^{2}}\right) / 2$. The points $z=0$ and $z=\infty$ correspond to the roots of the polynomial and are superattractors. The stability of the other fixed points (called strange fixed points) is established in the following results.

Proposition $2.1([?])$. The fixed point $z=1$ satisfies the following statements:

1. If $\left|\alpha-\frac{13}{6}\right|<\frac{1}{3}$, then $z=1$ is an attractor and, in particular, it is a superattractor for $\alpha=2$.

2. If $\left|\alpha-\frac{13}{6}\right|=\frac{1}{3}$, then $z=1$ is an indifferent point.

3. If $\left|\alpha-\frac{13}{6}\right|>\frac{1}{3}$, then $z=1$ is a repulsive fixed point.

Proposition 2.2 ([?]). The fixed points $z=s_{i}, i=1,2$, satisfy the following statements:

i) If $|\alpha-3|<\frac{1}{2}$, then $s_{1}$ and $s_{2}$ are attracting fixed points. In particular, for $\alpha=3$, $s_{1}$ and $s_{2}$ are superattractors.

ii) If $|\alpha-3|=\frac{1}{2}$, then $s_{1}$ and $s_{2}$ are indifferent points. In particular, for $\alpha=\frac{5}{2}, s_{1}=s_{2}=1$.

iii) If $|\alpha-3|>\frac{1}{2}$, then $s_{1}$ and $s_{2}$ are repulsive fixed points.

On the other hand, the derivative of this operator,

$$
O_{\alpha}^{\prime}(z)=2 z^{2} \frac{3(1-\alpha)+2 z\left(3-4 \alpha+2 \alpha^{2}\right)+3 z^{2}(1-\alpha)}{(1-2(\alpha-1) z)^{2}},
$$

allows us to calculate the critical points of $O_{\alpha}(z)$. The equation $O_{\alpha}^{\prime}(z)=0$ provides $z=0$, $z=\infty$ and

$$
z=\frac{3-4 \alpha+2 \alpha^{2} \pm \sqrt{-6 \alpha+19 \alpha^{2}-16 \alpha^{3}+4 \alpha^{4}}}{3(\alpha-1)}
$$

which are denoted by $c_{+}$and $c_{-}$, respectively. The orbits of the free critical points control the existence of Fatou components other than the basins of attraction of $z=0$ and $z=\infty$ (c.f. [?]).

The study of the parameter space enables us to analyse the dynamics of the rational function associated to an iterative method. Each point of the parameter space is associated to a complex value of $\alpha$, i.e. to a member of the family. Moreover, every value of $\alpha$ belonging to the same connected component of the parameter space gives rise to subsets of schemes of family with similar dynamical behaviour. 


\section{Critical points and the parameter space}

In this section we describe the positions of the critical points as well as the dynamical relation that their orbits posses. Critical points are crucial to analyze the parameter space since every periodic connected component of the Fatou set has at least a critical point related to it (c.f. [?]). In particular, all basins of attraction contain at least a critical point whose orbit converges to the attracting (or rationally indifferent) periodic orbit. The critical points of the operator $O_{\alpha}(z)$, i.e. the zeros of $O_{\alpha}^{\prime}(z)$, are given in Equation (4) and denoted by $c_{+}$and $c_{-}$,

$$
c_{ \pm}=\frac{3-4 \alpha+2 \alpha^{2} \pm \sqrt{-6 \alpha+19 \alpha^{2}-16 \alpha^{3}+4 \alpha^{4}}}{3(\alpha-1)} .
$$

We want to emphasize that, since we are working with complex numbers, we need to choose a branch of the square root. We have two possible solutions for the square root given by $a+b i$ and $-a-b i$, where $a, b \in \mathbb{R}$. As usual, if $a \neq 0$ we choose the value of the square root so that the real part is positive. If $a=0$ we choose the value so that the imaginary part is positive. The value of the square root changes when $-6 \alpha+19 \alpha^{2}-16 \alpha^{3}+4 \alpha^{4}$ is a real negative number. If we write $\alpha=x+i y$, it can be shown that this change on the determination takes place along the hyperbola $(x-1)^{2}-y^{2}=\frac{5}{8}$.

The changes on the determination of the critical points are an inconvenient when trying to study the dynamics of these maps because of the unboundedness of the hyperbolas. To avoid this problem we can use the property that $c_{+}=1 / c_{-}$and choose the one with greater modulus. On the other hand, it can be shown that $\left|c_{+}\right|=\left|c_{-}\right|=1$ on the circle $(x-1)^{2}+y^{2}=\frac{1}{2}$ and the segments $[0,1 / 2],[3 / 2,2]$. Hence, it makes sense to rename the critical point as follows. We denote by $c_{2}$ the critical point with modulus greater or equal than 1 and by $c_{1}$ the other critical point, i.e., $c_{2}=c_{+}$if $\left|c_{+}\right| \geq\left|c_{-}\right|$and $c_{2}=c_{-}$otherwise. This definition of the critical points will be necessary for studying the boundedness of parameters with bad dynamical behaviour in the next section.

As the following lemma shows, the dynamics of both critical points are not independent, being that the map $1 / z$ conjugates $O_{\alpha}(z)$ with itself.

Lemma 3.1. Let $I(z)=1 / z$. Then, fixed any $\alpha \in \mathbb{C}$ and $z \in \widehat{\mathbb{C}}, z \neq 0$, we have that

$$
O_{\alpha} \circ I(z)=I \circ O_{\alpha}(z)
$$

Proof.

$$
O_{\alpha} \circ I(z)=\left(\frac{1}{z}\right)^{3} \frac{\frac{1}{z}-2(\alpha-1)}{1-2(\alpha-1) \frac{1}{z}} \cdot \frac{z}{z}=\left(\frac{1}{z}\right)^{3} \frac{1-2(\alpha-1) z}{z-2(\alpha-1)}=\frac{1}{z^{3} \frac{z-2(\alpha-1)}{1-2(\alpha-1) z}}=I \circ O_{\alpha}(z) .
$$

The importance of this lemma comes from the fact that it ties the dynamics of all pairs of points $v$ and $w$ such that $v=1 / w$. Indeed, it follows from the lemma that $O_{\alpha}(v)=O_{\alpha}(I(w))=$ $I\left(O_{\alpha}(w)\right)=1 / O_{\alpha}(w)$. Iterating the argument we have that $O_{\alpha}^{n}(v)=1 / O_{\alpha}^{n}(w)$, for all $n \in \mathbb{N}$. In particular, we can apply this property to the critical points since $c_{2}=1 / c_{1}\left(c_{+}=1 / c_{-}\right)$. We can conclude that if one critical orbit converges to $z=0$ then the other one converges to $z=\infty$. This implies that it is enough to analyze the asymptotic behaviour of one of the critical 
orbits, i.e. the orbits of the critical points, to study the existence of any attractor other than the basins of attraction of the roots.

Once we have fixed the notation for the critical points and established the symmetry given by $1 / z$ we can analyse the parameter plane. In Figure 1 we plot a representation of it for $\operatorname{Re}(\alpha) \in(-0.4,4.6)$ and $\operatorname{Im}(\alpha) \in(-2,2)$. To do so, we use a $\mathrm{C}$ program. We create a grid of $1500 \times 1200$ points and associate a parameter to each point of the grid. We iterate the critical point $c_{+}$up to 150 times. If after fewer than 150 iterations the point $w$ is close enough to $z=0$ or $z=\infty\left(|w|<10^{-4}\right.$ or $\left.|w|>10^{4}\right)$ then we conclude that the critical orbit converges to one of the attractors which come from the roots of the polynomial and plot the parameter using a scaling from pallid blue to green to yellow and to red depending on the number of iterates taken before escaping. In this way we may observe level curves in the red region that delimit the sets of parameters which fall into the neighbourhoods of $z=0$ and $z=\infty$ given by $z$ such that $|z|<10^{-4}$ or $|z|>10^{4}$, respectively, in $m$ iterates. If after 150 iterates the orbit has not escaped to $z=0$ or $z=\infty$ then we plot the parameter in black. Black parameters are those ones for which we may have attractors that do not correspond to the roots and other dynamics which are not good for the convergence of the numerical method. We want to point out that if instead of $10^{-4}$ and $10^{4}$ we had used other tolerances $t_{o l}$ and $t_{o l}$ such that $t o l_{\epsilon} \neq 1 /$ tol $_{\infty}$ then we could observe the hyperbola where the definition of the square root changes in the representation of the parameter space. Indeed, we would observe this hyperbola as a discontinuity in the level curves which tell us how fast the critical point converges to $z=0$ or $z=\infty$ given a parameter $\alpha$. Since we take symmetric tolerances, these discontinuities can not be observed due to the symmetry in the dynamical plane explained in Lemma 3.1.

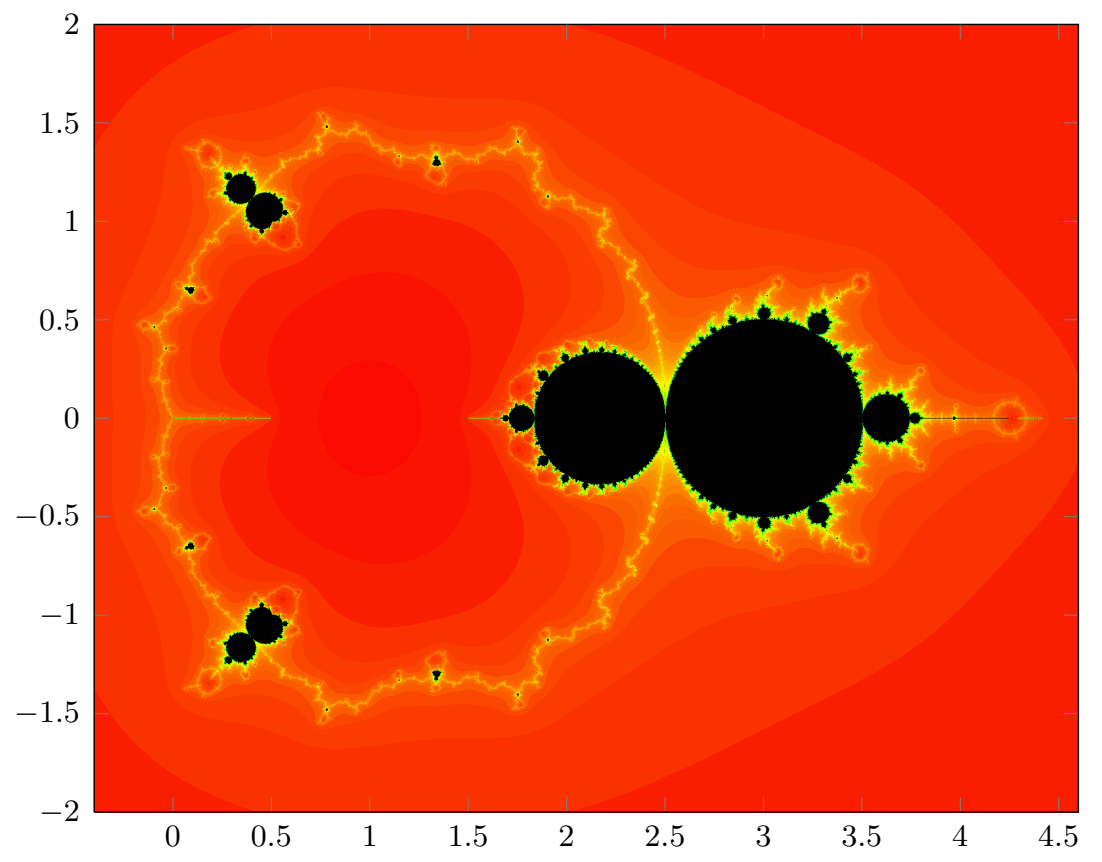

Figure 1: Parameter space of the family $O_{\alpha}(z)$.

In Figure 1 we observe the boundedness locus in the parameter space, called the Cat set. It corresponds to the set of parameters $\alpha$ such that the critical orbits do not converge to $z=0$ or $z=\infty$. The Cat set contains two big disks corresponding to the $\alpha$ values for which the fixed points $z=1$ (the head, $\left|\alpha-\frac{13}{6}\right|<\frac{1}{3}$ ) and $s_{1}$ and $s_{2}$ (the body, $|\alpha-3|<\frac{1}{2}$ ) become 
attracting. In that case, the critical points belong to the basins of attraction of the strange fixed points. The intersection point of the boundaries of the head and the body of the Cat set corresponds to $\alpha=\frac{5}{2}$. The boundary of the Cat set is exactly the set of parameters for which the dynamics changes abruptly under small changes of $\alpha$, i.e. it is the bifurcation locus of the family of Chebyshev-Halley acting on the polynomials $z^{2}+c$ (see [?]).

\section{The Cat set is bounded}

The goal of this section is to give an explicit bound for the Cat set. Recall that it corresponds to the parameters $\alpha$ for which the free critical points $c_{1}$ and $c_{2}$ do not converge under iteration to the superattracting fixed points $z=0$ and $z=\infty$, which correspond to the zeros of the quadratic polynomial. Hence, it consists of the parameters $\alpha$ for which there might be stable behaviour other than the basins of attraction of the roots and there might be an open set of initial conditions where the numerical algorithm fails.

The main result of the section is the following theorem. It tells us that the Cat set is bounded and provides a criterion which guarantees the stability of the numerical algorithm.

Theorem 4.1. If $|\alpha|>8.57$ then the critical point $c_{2}$ belongs to $\mathcal{A}(\infty)$. Consequently, if $|\alpha|>8.57$ then $\alpha$ does not belong to the Cat set.

It follows from the theorem that if $|\alpha|>8.57$ then $\alpha$ does not belong to the Cat set. However, we want to remark that numerical experiments show that this bound is not sharp (c.f. Figure 1). On the other hand, these numerical experiments rely on the bound since it tells us how far we need to look for to find all bad dynamical behaviour.

Along this section it will be convenient to work with the parameter $a=2(\alpha-1)$ instead of $\alpha$ in order to simplify calculus. The proof of Theorem 4.1 will follow directly from Proposition 4.6, where we prove the result for the parameter $a$. With this change of parameters the maps $O_{\alpha}(z)$ (Equation (2)) can be written as

$$
O_{a}(z)=z^{3} \frac{z-a}{1-a z}
$$

and the new formula for the critical points is

$$
c_{ \pm}:=c_{ \pm}(a):=\frac{1}{3 a}\left(2+a^{2} \pm \sqrt{\left(a^{2}-4\right)\left(a^{2}-1\right)}\right) .
$$

Recall from Section 3 that we denote $c_{2}=c_{+}$and $c_{1}=c_{-}$if $\left|c_{+}\right| \geq\left|c_{-}\right|$. Otherwise we denote $c_{2}=c_{-}$and $c_{1}=c_{+}$.

We want to point out that the fact that the Cat set is bounded had already been proven in [?]. In the preliminaries they study the family $\left.G_{a, b}(z)=b z^{3}(z-a) / 1-a z\right)$, which coincides with the family $O_{a}(z)$ for $b=1$. It follows from their work that, fixed $b=1$, there exists a constant $M$ such that if $|a|>M$ then $c_{2}$ belongs in $A(\infty)$. However, they provide no estimation of how large this constant $M$ may be. We provide this estimation in Proposition 4.6. Before proving it we introduce some auxiliary results.

Lemma 4.2. For any two complex numbers $u$ and $v$ such that $|v| \neq 0$, then either $|u+v|>|u|$ or $|u-v|>|u|$. 
Proof. Let $u=x_{1}+y_{1} i$ and $v=x_{2}+y_{2} i$. If $|u+v| \leq|u|$ then, by developing both moduli, $|v|^{2} \leq-2\left(x_{1} x_{2}+y_{1} y_{2}\right)$. This inequality allows us to write $2|v|^{2}+|u|^{2} \leq|u-v|^{2}$. Then, $|u|^{2}<|u-v|^{2}$ and we obtain that $|u-v|>|u|$.

Next result follows directly from the previous lemma since $c_{2}$ is taken to be the critical point with greater modulus.

Corollary 4.3. The critical point $c_{2}$ satisfies $\left|c_{2}\right|>\left|a^{2}+2\right| / 3|a|$.

The following equalities are used in the proof of Proposition 4.6.

Lemma 4.4. The following statements are satisfied:
1. $c_{2}^{2}=\frac{2\left(a^{2}+2\right) c_{2}}{3 a}-1$
2. $\frac{c_{2}-a}{1-a c_{2}}=3 c_{1}-\frac{4}{a}$.

Proof. The first statement is easily proved:

$$
\begin{gathered}
c_{ \pm}^{2}=\frac{\left(a^{2}+2 \pm \sqrt{\left(a^{2}-1\right)\left(a^{2}-4\right)}\right)^{2}}{9 a^{2}}= \\
=\frac{2\left(a^{2}+2\right)\left(a^{2}+2 \pm \sqrt{\left(a^{2}-1\right)\left(a^{2}-4\right)}\right)}{9 a^{2}}-1=\frac{2\left(a^{2}+2\right) c_{ \pm}}{3 a}-1 .
\end{gathered}
$$

To prove the second statement we have:

$$
\begin{gathered}
\frac{c_{+}-a}{1-a c_{+}}=\frac{\frac{a^{2}+2+\sqrt{\left(a^{2}-1\right)\left(a^{2}-4\right)}}{3 a}-a}{1-a \frac{a^{2}+2+\sqrt{\left(a^{2}-1\right)\left(a^{2}-4\right)}}{3 a}}= \\
=\frac{a^{2}+2+\sqrt{\left(a^{2}-1\right)\left(a^{2}-4\right)}-3 a^{2}}{3 a-a\left(a^{2}+2+\sqrt{\left(a^{2}-1\right)\left(a^{2}-4\right)}\right)}=\frac{2\left(1-a^{2}\right)+\sqrt{\left(a^{2}-1\right)\left(a^{2}-4\right)}}{a\left(1-a^{2}-\sqrt{\left(a^{2}-1\right)\left(a^{2}-4\right)}\right)}= \\
=\frac{\left(2\left(1-a^{2}\right)+\sqrt{\left(a^{2}-1\right)\left(a^{2}-4\right)}\right)\left(1-a^{2}+\sqrt{\left(a^{2}-1\right)\left(a^{2}-4\right)}\right)}{a\left(\left(1-a^{2}\right)^{2}-\left(a^{2}-1\right)\left(a^{2}-4\right)\right)}= \\
=\frac{2\left(1-a^{2}\right)^{2}+3\left(1-a^{2}\right) \sqrt{\left(a^{2}-1\right)\left(a^{2}-4\right)}+\left(a^{2}-1\right)\left(a^{2}-4\right)}{3 a\left(a^{2}-1\right)}= \\
=\frac{3\left(a^{2}-1\right)\left(a^{2}-2\right)-3\left(a^{2}-1\right) \sqrt{\left(a^{2}-1\right)\left(a^{2}-4\right)}}{3 a\left(a^{2}-1\right)}= \\
=\frac{a^{2}-2-\sqrt{\left(a^{2}-1\right)\left(a^{2}-4\right)}}{a}=3 c_{-}-\frac{4}{a} .
\end{gathered}
$$

Similarly, $\frac{c_{-}-a}{1-a c_{-}}=3 c_{+}-\frac{4}{a}$. 
Next proposition is the main result used in the proof of Proposition 4.6 (and Theorem 4.1). It gives us a lower bound depending on $|a|$ which guarantees that a point $z$ belongs to $\mathcal{A}(\infty)$. We want to point out that by using Lemma 3.1 we can also obtain a bound which guarantees that a point $z \in \mathcal{A}(0)$. Indeed, it follows from this lemma that if a point $z \in \mathcal{A}(\infty)$ then $1 / z \in \mathcal{A}(0)$.

Proposition 4.5. If $|z|>\lambda(|a|+1)$, where $\lambda>1$, then $z \in \mathcal{A}(\infty)$.

Proof. As $|z|>\lambda(|a|+1)$ and $\lambda>1$ we have

$$
|z-a| \geq|| z|-| a||=|z|-|a|>\lambda(|a|+1)-|a|>\lambda(|a|+1)-\lambda|a|=\lambda
$$

and

$$
|1-a z|<1+|a z|<|z|+|a||z|=|z|(1+|a|)<|z|^{2} .
$$

Then,

$$
\left|O_{a}(z)\right|=|z|^{3}\left|\frac{z-a}{1-a z}\right|>|z|^{3} \frac{\lambda}{|z|^{2}}=\lambda|z| .
$$

Now assume that $|z|>\lambda(|a|+1)$. Then, $\left|O_{a}(z)\right|>\lambda|z|$ and $\left|O_{a}^{n}(z)\right|>\lambda^{n}|z|$, where $\lambda>1$ and $n \in \mathbb{N}$. We can conclude that if $\left|O_{a}(z)\right|>\lambda(|a|+1)$ then $O_{a}^{n}(z) \rightarrow \infty$ as $n \rightarrow \infty$ and $z \in \mathcal{A}(\infty)$.

From these results we calculate a bound that assures that $c_{2}$ belongs to $\mathcal{A}(\infty)$.

Proposition 4.6. For $|a|>15.133$ the critical point $c_{2}$ is in the basin of attraction of $\infty$.

Proof. It follows from Lemma 4.5 that, if $\left|O_{a}\left(c_{2}\right)\right|>\lambda(|a|+1)$ for some $\lambda>1$, then $c_{2} \in \mathcal{A}(\infty)$. Notice that it suffices to prove $\left|O_{a}\left(c_{2}\right)\right|>|a|+1$ in order to apply this lemma. In the following, we show that if $|a|>15.133$ then $\left|O_{a}\left(c_{2}\right)\right|>|a|+1$.

We first provide an alternative expression for $\left|O_{a}\left(c_{2}\right)\right|$ using the equalities introduced in Lemma 4.4.

$$
\begin{gathered}
\left|O_{a}\left(c_{2}\right)\right|=\left|c_{2}\right|^{3}\left|\frac{c_{2}-a}{1-a c_{2}}\right|=\left|c_{2}\right|^{3}\left|3 c_{1}-\frac{4}{a}\right|=\left|c_{2}\right|\left|3 c_{2}-\frac{4 c_{2}^{2}}{a}\right|= \\
=\left|c_{2}\right|\left|3 c_{2}-\frac{4}{a}\left(\frac{2\left(a^{2}+2\right) c_{2}}{3 a}-1\right)\right|=\frac{1}{3|a|^{2}}\left|c_{2}\right|\left|c_{2}\left(a^{2}-16\right)+12 a\right|= \\
=\frac{1}{3|a|^{2}}\left|c_{2}^{2}\left(a^{2}-16\right)+12 a c_{2}\right|=\frac{1}{3|a|^{2}}\left|\left(\frac{2\left(a^{2}+2\right) c_{2}}{3 a}-1\right)\left(a^{2}-16\right)+12 a c_{2}\right|= \\
=\frac{1}{9} \frac{\left|2 c_{2} a^{4}-3 a^{3}+8 c_{2} a^{2}+48 a-64 c_{2}\right|}{|a|^{3}}=\frac{1}{9} \frac{\left|2\left(a^{4}+4 a^{2}-32\right) c_{2}-3 a\left(a^{2}-16\right)\right|}{|a|^{3}} .
\end{gathered}
$$

The modulus of the numerator verifies

$$
\left|2\left(a^{4}+4 a^{2}-32\right) c_{2}-3 a\left(a^{2}-16\right)\right|>|2| a^{4}+4 a^{2}-32|| c_{2}|-3| a|| a^{2}-16|| .
$$

Moreover, we have the equality

$$
|2| a^{4}+4 a^{2}-32|| c_{2}|-3| a|| a^{2}-16||=2\left|a^{4}+4 a^{2}-32\right|\left|c_{2}\right|-3|a|\left|a^{2}-16\right|
$$

if $c_{2}$ satisfies 


$$
\left|c_{2}\right|>\frac{3|a|\left|a^{2}-16\right|}{2\left|a^{4}+4 a^{2}-32\right|} .
$$

To obtain the parameters for which the previous holds we use the intermediate inequality

$$
\left|c_{2}\right|>\frac{3|a|\left(|a|^{2}+16\right)}{2\left(|a|^{4}-4|a|^{2}-32\right)}>\frac{3|a|\left|a^{2}-16\right|}{2\left|a^{4}+4 a^{2}-32\right|} .
$$

By definition of $c_{2}$ we have $\left|c_{2}\right| \geq 1$. Moreover, the inequality

$$
1>\frac{3|a|\left(|a|^{2}+16\right)}{2\left(|a|^{4}-4|a|^{2}-32\right)}
$$

is satisfied for $|a|>4.23$. Therefore, then first inequality in (8) holds for $|a|>4.23$.

Similarly, to prove the second inequality in (8), we observe that $|a|^{4}>4|a|^{2}+32$ is satisfied for $|a|>2 \sqrt{2}$. M oreover, $4|a|^{2}+32>\left|4 a^{2}-32\right|$ and then

$$
\left|a^{4}+4 a^{2}-32\right|>|a|^{4}-\left|4 a^{2}-32\right|>|a|^{4}-4|a|^{2}-32 .
$$

We conclude that the inequality (7) is satisfied for $|a|>4.23$. Up to here we have proven that, for $|a|>4.23$, we have the inequality

$$
\left|O_{a}\left(c_{2}\right)\right|>\frac{2\left|a^{4}+4 a^{2}-32\right|\left|c_{2}\right|-3|a|\left|a^{2}-16\right|}{9|a|^{3}} .
$$

As we want to show that $\left|O_{a}\left(c_{2}\right)\right|>|a|+1$ we have to prove the inequality

$$
2\left|a^{4}+4 a^{2}-32\right|\left|c_{2}\right|-3|a|\left|a^{2}-16\right|>9\left|a^{3}\right|(|a|+1)
$$

or equivalently

$$
\left|c_{2}\right|>\frac{9|a|^{3}(|a|+1)+3|a|\left|a^{2}-16\right|}{2\left|a^{4}+4 a^{2}-32\right|} .
$$

To simplify computations we divide the previous inequality into the following

$$
\begin{array}{r}
\left|c_{2}\right|>5, \\
\frac{9|a|^{3}(|a|+1)+3|a|\left|a^{2}-16\right|}{2\left|a^{4}+4 a^{2}-32\right|}<5,
\end{array}
$$

and determine in which range of parameters they are both satisfied. We begin with Equation (12). We have

$$
\begin{gathered}
9|a|^{3}(|a|+1)+3|a|\left|a^{2}-16\right|<9|a|^{4}+12|a|^{3}+48|a|< \\
<10\left(|a|^{4}-4|a|^{2}-32\right)<10\left|a^{4}+4 a^{2}-32\right|,
\end{gathered}
$$

where the third inequality corresponds to Equation $(9)$ and is verified for $|a|>2 \sqrt{2}$ and the second inequality

$$
9|a|^{4}+12|a|^{3}+48|a|<10\left(|a|^{4}-4|a|^{2}-32\right)
$$

is verified if

$$
|a|^{4}-12|a|^{3}-40|a|^{2}-48|a|-320>0,
$$


which holds for $|a|>14.98$.

For Equation (11), it follows from Corollary 4.3 that

$$
\left|c_{2}\right|>\frac{\left|a^{2}+2\right|}{3|a|}
$$

and we have $\left|a^{2}+2\right|>\left|a^{2}\right|-2>15|a|$ if $|a|>15.133$. Hence, $\left|c_{2}\right|>5$ if $|a|>15.133$. Then, the proposition is satisfied for $|a|>15.133$.

From this result, Theorem 4.1 is proved using that $\alpha=a / 2+1$. Therefore, we can assure that, for $|\alpha|>8.57$, the Fatou set of the operator (2) is the union of the basins of attraction of 0 and $\infty$, that is, almost every initial condition yields to one of the roots of the polynomial.

\section{The boundaries of the head and the body of the Cat}

In this section we analyse the dynamics of the map $O_{\alpha}(z)$ on the boundaries given in Proposition 2.1 and Proposition 2.2, respectively, i.e. for values of parameters $\alpha$ such that $\left|\alpha-\frac{13}{6}\right|=\frac{1}{3}$ and $|\alpha-3|=\frac{1}{2}$. For these parameters we have indifferent fixed points, i.e. points $z_{0} \in \widehat{\mathbb{C}}$ such that $O_{\alpha}\left(z_{0}\right)=z_{0}$ and $\left|O_{\alpha}^{\prime}\left(z_{0}\right)\right|=1$.

The following proposition tells us that the point $z=1$ takes all multipliers in $\mathbb{S}^{1}$ for $\alpha$ in the circle of parameters of center $13 / 6$ and radius $1 / 3$.

Proposition 5.1. The fixed point $z=1$ has multiplier $e^{2 \pi i \rho}$ if and only if $\alpha=\frac{13}{6}+\frac{1-2 e^{2 \pi i \rho}}{3\left(e^{2 \pi i \rho}-2\right)}$.

Proof. From $O_{\alpha}^{\prime}(z)$ we know that $z=1$ is an indifferent fixed point for all values of $\alpha$ belonging to the boundary of the head, $\alpha_{\theta}=\frac{13}{6}+\frac{1}{3} e^{i \theta}, \theta \in[0,2 \pi)$ :

$$
O_{\alpha_{\theta}}^{\prime}(1)=\frac{2 e^{i \theta}+1}{2+e^{i \theta}} \text { and }\left|O_{\alpha_{\theta}}^{\prime}(1)\right|=1
$$

The multiplier $O_{\alpha_{\theta}}^{\prime}(1)$ restricted to the boundary of the head takes all values in the unit circle exactly once. Indeed, if we parametrize the unit circle by $e^{2 \pi i \rho}$ with $\rho \in[0,1)$, the fixed point $z=1$ has multiplier $e^{2 \pi i \rho}$ when $O_{\alpha_{\theta}}(1)=e^{2 \pi i \rho}$, that implies $\alpha=13 / 6+\left(1-2 e^{2 \pi i \rho}\right) /\left(3\left(e^{2 \pi i \rho}-2\right)\right)$, where $\left(1-2 e^{2 \pi i \rho}\right) /\left(e^{2 \pi i \rho}-2\right)=e^{i \theta} \in \mathbb{S}^{1}$.

Analogously, $z=s_{1}, s_{2}$ are indifferent fixed points for all values of $\alpha$ belonging to the boundary of the body.

Proposition 5.2. The fixed points $z=s_{1}, s_{2}$ have multiplier $e^{2 \pi i \rho}$ if and only $\alpha=3+e^{\pi i(2 \rho+1)} / 2$.

Proof. The fixed points $s_{1}$ and $s_{2}$ are indifferent on the boundary of the body, that is for $\alpha_{\theta}=3+\frac{1}{2} e^{i \theta}, \theta \in[0,2 \pi)$ :

$$
O_{\alpha_{\theta}}^{\prime}\left(s_{1}\right)=O_{\alpha_{\theta}}^{\prime}\left(s_{2}\right)=-e^{i \theta} \text { and }\left|O_{\alpha_{\theta}}^{\prime}\left(s_{1}\right)\right|=\left|O_{\alpha_{\theta}}^{\prime}\left(s_{2}\right)\right|=1 .
$$

The multiplier $O_{\alpha_{\theta}}^{\prime}\left(s_{1,2}\right)$ restricted to the boundary of the head takes all values in the unit circle exactly once. The points $s_{1}$ and $s_{2}$ have multiplier $e^{2 \pi i \rho}$, with $\rho \in[0,1)$, when $O_{\alpha_{\theta}}^{\prime}\left(s_{1,2}\right)=e^{2 \pi i \rho}$. It implies $\alpha=3+e^{\pi i(2 \rho+1)} / 2$. 
The dynamics of the operators for parameters in these two circles can be very different depending on whether the indifferent fixed points are rational or irrational. We will first describe the rational case. It follows from the discussion at the beginning of the section that, given any rational number $p / q \in[0,1)$ with $p, q \in \mathbb{N}$ coprime, there exists a unique parameter $\alpha$ in the boundary of the head and a unique parameter $\alpha$ in the boundary of the body of the cat set for which the corresponding fixed points have multiplier $e^{2 \pi i p / q}$. It follows from the Leau-Fatou flower theorem (see [?]) that the corresponding rationally indifferent fixed point have $q$-cycles of parabolic basins of points which are attracted under iteration to the fixed point. It is also known that each of these cycles of parabolic components contains at least one critical point. In Figure 2 we plot the dynamical plane of the parameter $\alpha=13 / 6+\left(1-2 e^{2 \pi i / 3}\right) /\left(3\left(e^{2 \pi i / 3}-2\right)\right.$ on the boundary of the head. For this parameter, the fixed point $z=1$ is rationally indifferent with multiplier $e^{2 \pi i 1 / 3}$. We also plot the orbits of the critical points $c_{2}$ (white) and $c_{1}$ (red). They belong to two different cycles of parabolic basins of period 3. However, the convergence to the fixed point is very slow and numerically it is very difficult to distinguish the different parabolic basins. In Figure 3 we plot the dynamical plane for $\alpha=3+e^{\pi i(2 / 3+1)} / 2$. In this case, the fixed points $s_{1}$ and $s_{2}$ (plotted as blue circles) have multiplier $e^{2 \pi i 1 / 3}$. Each of the rationally indifferent fixed points has a unique cycle of parabolic basins of period 3. We also plot the orbits of the critical points $c_{2}$ (white) and $c_{1}$ (red) and observe how the convergence to the fixed point is very slow. The figures are done using a $\mathrm{C}$ program similar to the one used for the parameter space. In this case we create a grid of points in the dynamical plane and iterate them. If the orbit of a point converges to $z=0\left(|w|<10^{-4}\right)$ in fewer than 150 iterates we plot the point in black. If it converges to infinity $\left(|w|>10^{4}\right)$ in fewer than 150 iterates we use the scaling from pallid blue to pallid green to pallid yellow and to red which was already used in the parameter space. Otherwise we assume that the orbit converges to a parabolic point and use the corresponding colours.

Given any irrational number $\rho \in[0,1) \cap(\mathbb{R} \backslash \mathbb{Q})$, there exists a unique parameter in the boundary of the head and a unique parameter in the boundary of the body for which the corresponding fixed points have multiplier $e^{2 \pi i \rho}$. These fixed points may be the center of Siegel disks. C. L. Siegel [?] proved that if the irrational number $\rho$ is diophantine then any irrational fixed point with multiplier $e^{2 \pi i \rho}$ is the center of a Siegel disk (this condition was improved later by A. D. Bryuno [?] for a bigger set of irrational numbers). In Figure 4 and Figure 5 we show the Siegel disks obtained when picking parameters in the boundary of the head and the body of the Cat set for which the corresponding fixed points have multiplier $e^{2 \pi i \rho}$ with $\rho$ equal to the golden number $(1+\sqrt{5}) / 2$, which is diophantine. The pictures are done using a modification of the $\mathrm{C}$ program used for the parabolic case. We draw the boundaries of the Siegel disks by plotting 4000 points of the orbit of the critical points (it is known that the orbit of at least a critical point accumulates on the boundary of a Siegel disk, c.f. [?]). We also plot 4000 points of the orbits of some points inside the Siegel disks to show how Siegel disks are foliated with invariant curves.

We finish the section with some comments about the decorations of the head and the body of the Cat set. They are surrounded with 'bulbs' in a similar way than the main Cardioid of the Mandelbrot set is. These bulbs contain parameters which yield to the appearance of attracting cycles. In the paper [?], the bulbs involving attracting cycles of period 2 are obtained. The parameters where the bulbs are joined with the head and the body of the Cat set are bifurcation points. They correspond to the parameters with rationally indifferent fixed points described in Proposition 5.1 and Proposition 5.2. If the joining parameter is such that there is a rationally indifferent fixed point of multiplier $e^{2 \pi i p / q}$ with $p, q \in \mathbb{N}$ coprime, then the corresponding bulb 

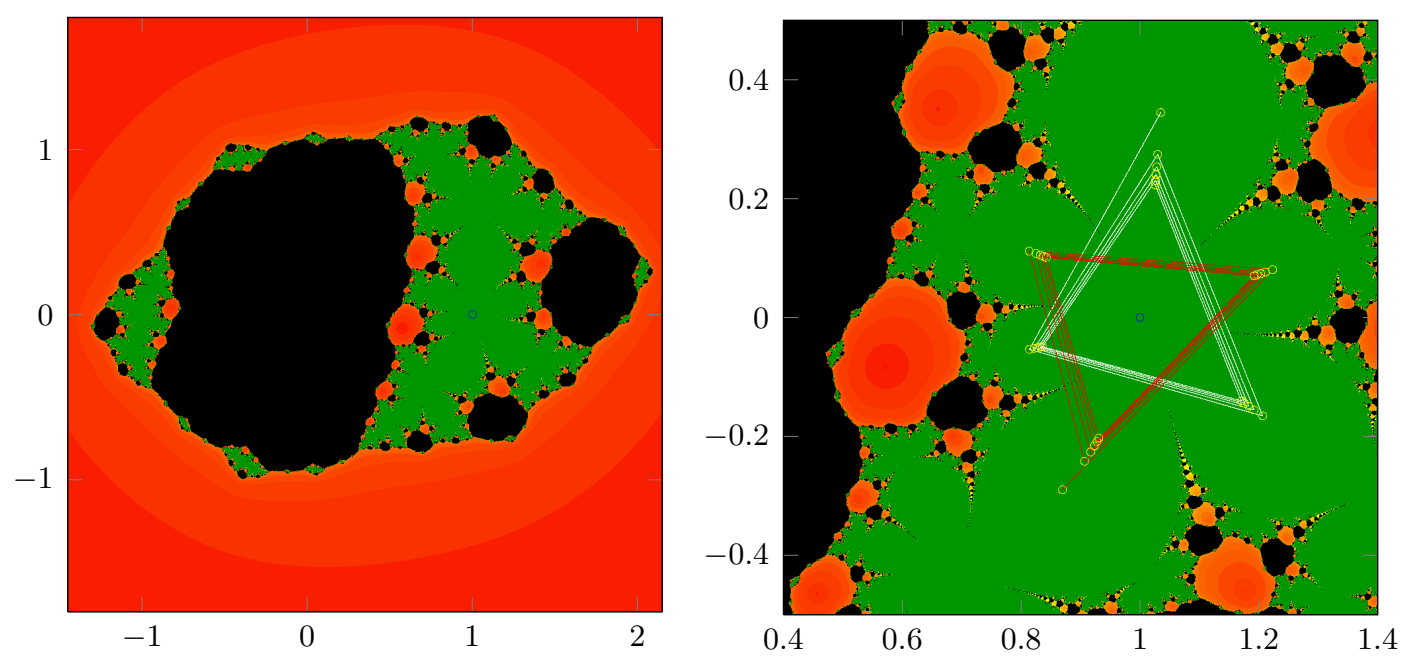

Figure 2: Dynamical plane of $O_{\alpha}(z)$ for $\alpha=13 / 6+\left(1-2 e^{2 \pi i / 3}\right) /\left(3\left(e^{2 \pi i / 3}-2\right)\right.$. For this parameter the fixed point $z=1$ has multiplier $e^{2 \pi i / 3}$. The figures have $900 \times 900$ points.
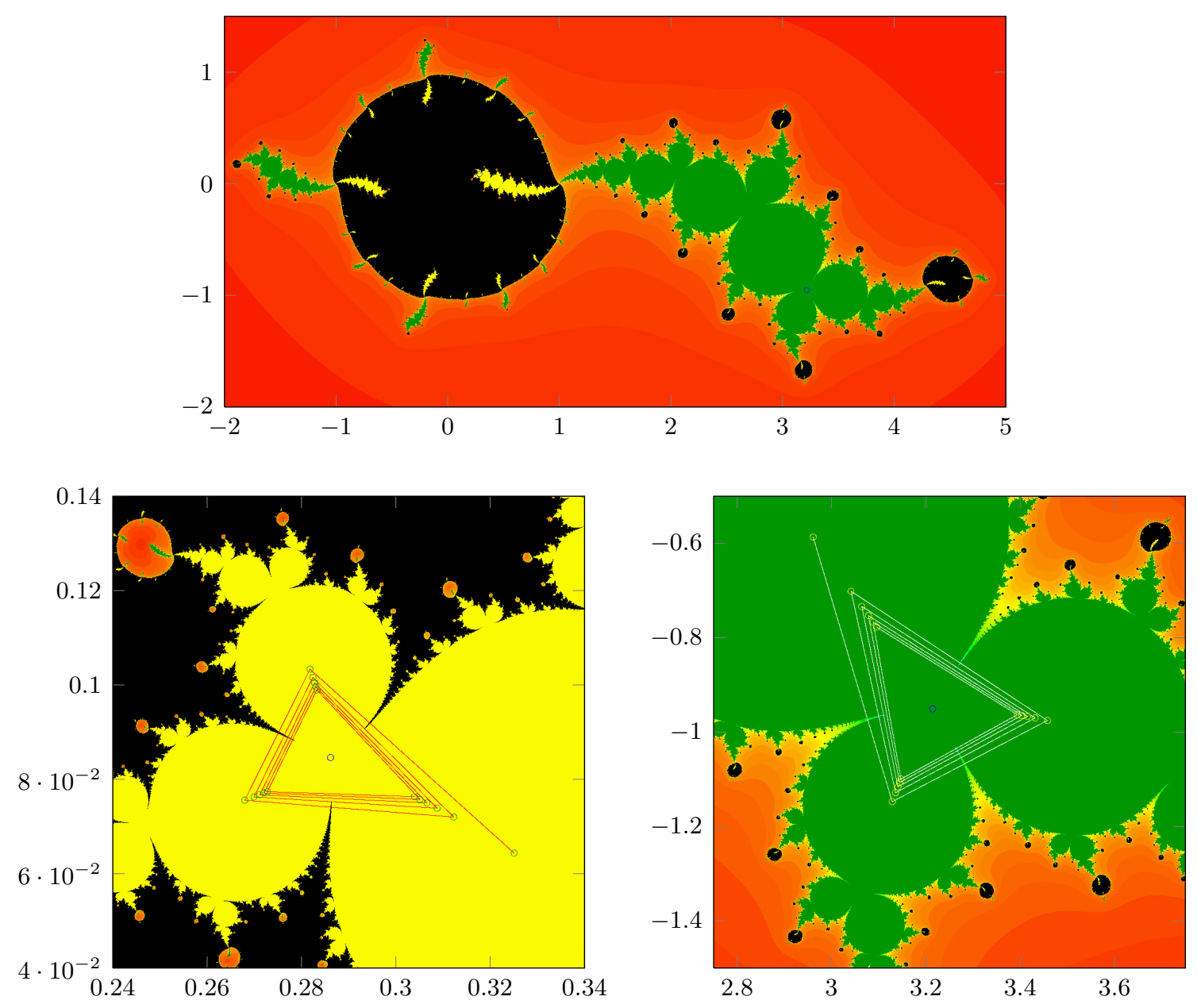

Figure 3: Dynamical plane of $O_{\alpha}(z)$ for $\alpha=3+e^{\pi i(2 / 3+1)} / 2$. For this parameter the strange fixed points have multiplier $-e^{\pi i(2 / 3+1)}=e^{2 \pi i / 3}$. The upper figure has $1800 \times 900$ points. The bottom figures have $900 \times 900$ points. 

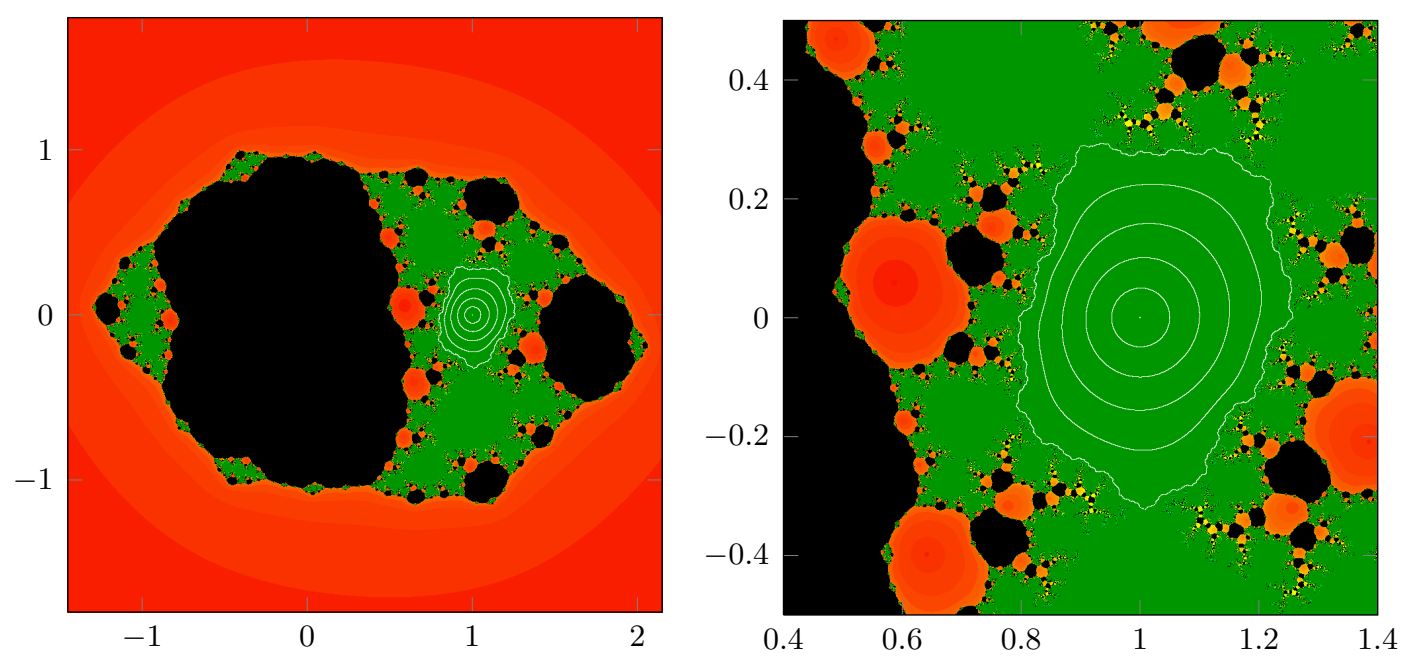

Figure 4: Dynamical plane of $O_{\alpha}(z)$ for $\alpha=13 / 6+\left(1-2 e^{\pi i(1+\sqrt{5})}\right) /\left(3\left(e^{\pi i(1+\sqrt{5})}-2\right)\right.$. The fixed point $z=1$ has multiplier $e^{2 \pi i \theta}$, where $\theta=(1+\sqrt{5}) / 2$. The figures have $900 \times 900$ points.
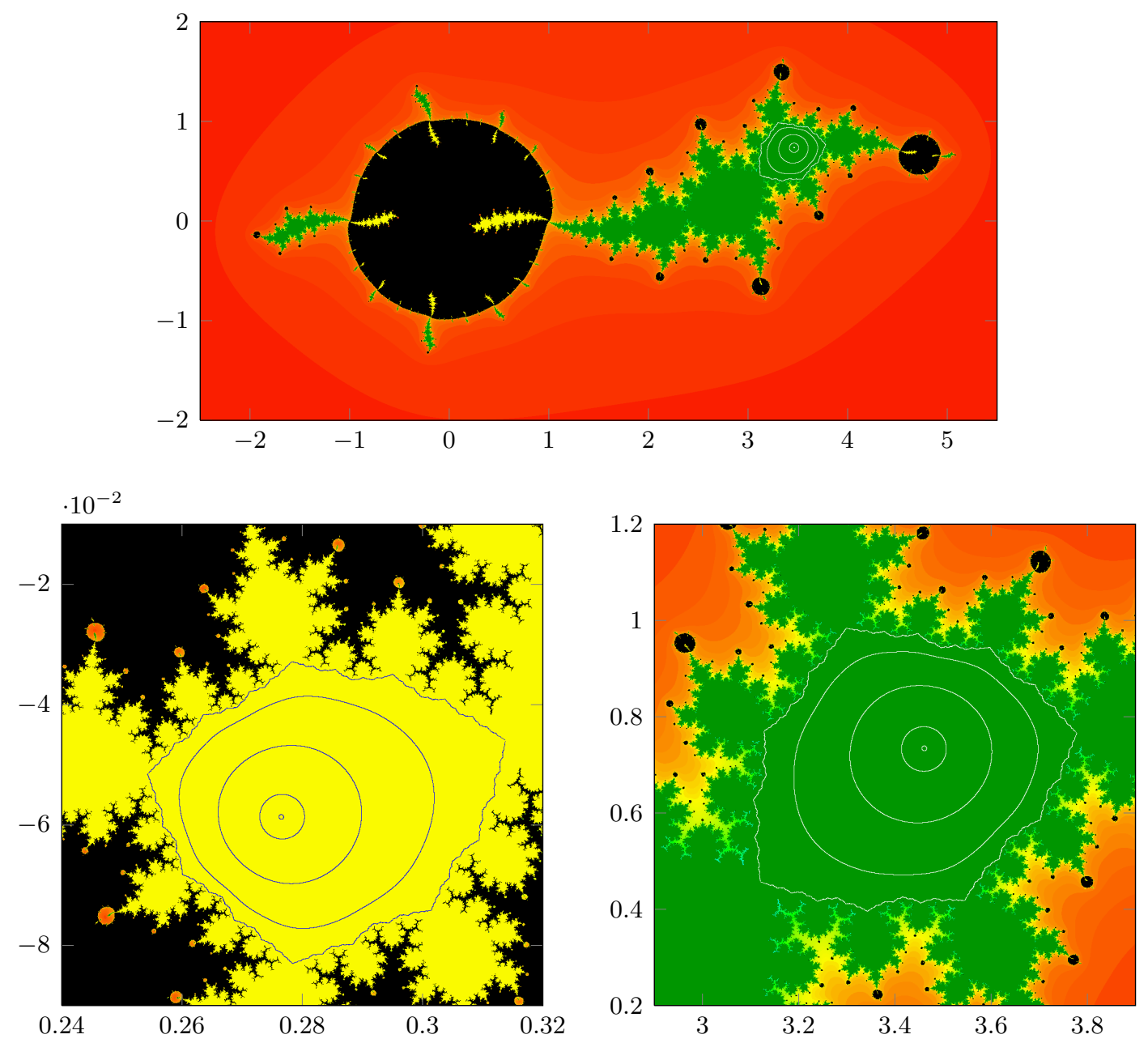

Figure 5: Dynamical plane of $O_{\alpha}(z)$ for $\alpha=3+e^{\pi i \sqrt{5}} / 2$. The strange fixed points have multiplier $-e^{\pi i \sqrt{5}}=e^{2 \pi i \theta}$, where $\theta=(1+\sqrt{5}) / 2$. The upper figure has $1800 \times 900$ points. The bottom figures have $900 \times 900$ points. 
is an open set of parameters for which there is an attracting cycle of period $q$ (see Figure 6). Moreover, the value $p$ can be used to describe the rotation within the points of the cycle.
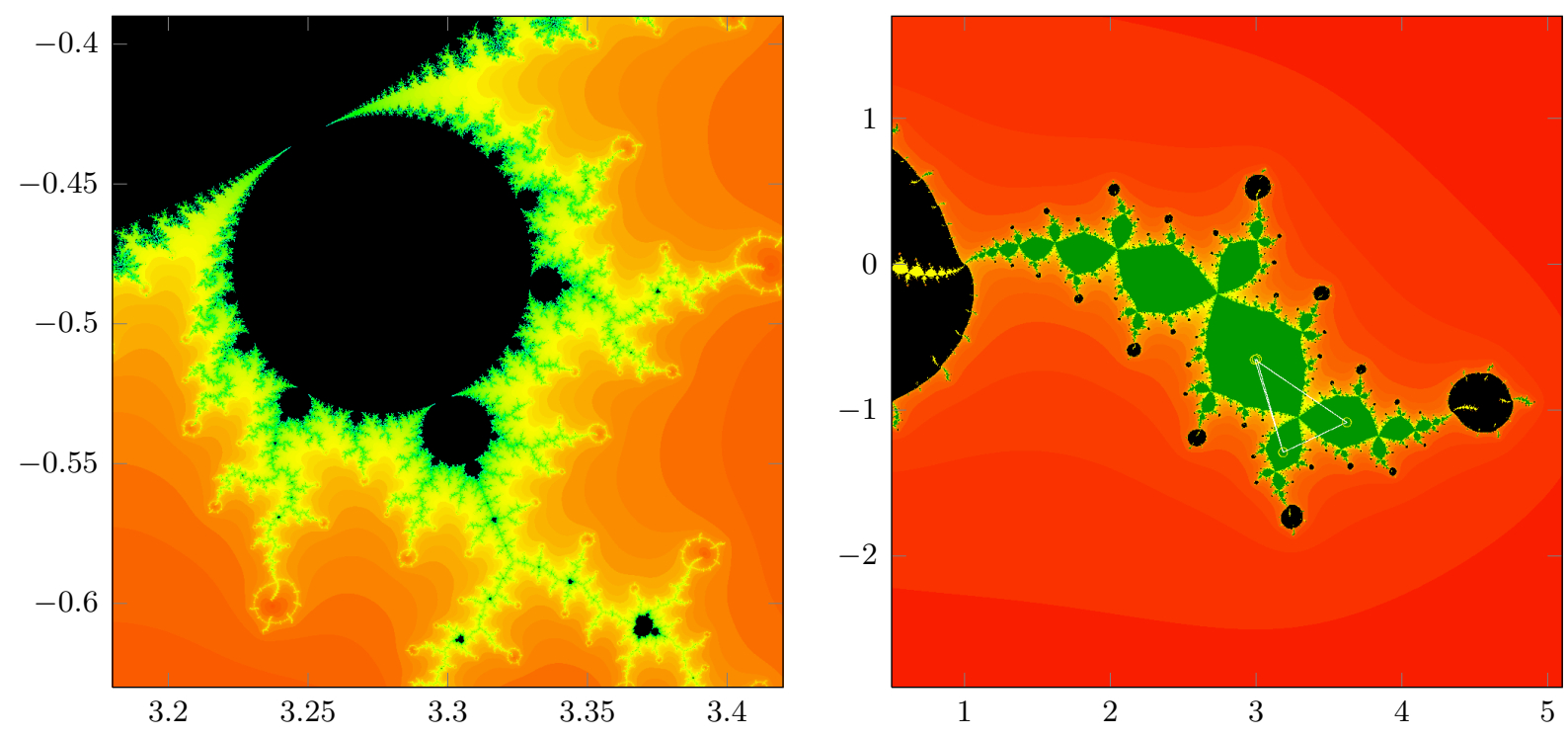

Figure 6: The left figure corresponds to the bulb which which shares the boundary parameter $\alpha=3+e^{\pi i(2 / 3+1)} / 2$ with the body of the Cat set. For this parameter the map $O_{\alpha}(z)$ has a parabolic point of multiplier $e^{2 \pi i 1 / 3}$ (see Figure 3). The right figure shows a period 3 attracting cycle for a parameter within this bulb $(\alpha=3.28-0.48 i)$. Both figures have $900 \times 900$ points.

\section{Dynamical study on degree $n$ polynomials}

The goal of this section is to provide numerical studies of how the family of Chebyshev-Halley type methods acts on the family of degree $n$ polynomials $z^{n}+c$. We will justify that, as it is the case with the operator studied above, it is enough to analyse the orbit of one critical point to determine if there is any stable behaviour other than the basins of attraction of the zeros of the polynomial.

We provide the operator applied to the polynomials $z^{n}-1$. This is enough since the conjugacy $\eta(z)=z / \sqrt[n]{-c}$, which sends the roots of $z^{n}+c$ to the roots of $z^{n}-1$, conjugates the operators obtained in each case, i.e. $O_{n, \alpha,-1}(z)=\eta \circ O_{n, \alpha, c} \circ \eta^{-1}(z)$ where $O_{n, \alpha, c}$ denotes the operator applied to $z^{n}+c$.

When applying the family of Chebyshev-Halley type methods to the polynomials $z^{n}-1$ we obtain the operator

$$
\begin{gathered}
O_{n, \alpha}(z)=z-\frac{\left(z^{n}-1\right)\left((-1+2 \alpha+n-2 \alpha n)+(1-2 \alpha-3 n+2 \alpha n) z^{n}\right)}{2 n z^{n-1}\left(\alpha(n-1)\left(z^{n}-1\right)-n z^{n}\right)}= \\
=\frac{(1-2 \alpha)(n-1)+\left(2-4 \alpha-4 n+6 \alpha n-2 \alpha n^{2}\right) z^{n}+(n-1)(1-2 \alpha-2 n+2 \alpha n) z^{2 n}}{2 \alpha n(1-n) z^{n-1}+2 n(-\alpha-n+\alpha n) z^{2 n-1}} .
\end{gathered}
$$

This operator has $2 n$ finite fixed points, i.e. solutions of $O_{n, \alpha}(z)=z$. Besides the $n t h$-roots of the unity, which are the zeros of the polynomial $z^{n}-1$ and are superattracting fixed points of the method, there are $n$ fixed points which are the solutions of 


$$
-1+2 \alpha+n-2 \alpha n+(1-2 \alpha-3 n+2 \alpha n) z^{n}=0 .
$$

The point $z=\infty$ is fixed since the term with bigger exponent on $z$ in the numerator is $(n-1)(1-2 \alpha-2 n+2 \alpha n) z^{2 n}$ and in the denominator is $2 n(-\alpha-n+\alpha n) z^{2 n-1}$. Therefore, the dynamics around $z=\infty$ is locally like

$$
\frac{(n-1)(1-2 \alpha-2 n+2 \alpha n)}{2 n(-\alpha-n+\alpha n)} z=: \lambda z .
$$

Moreover, $z=\infty$ is a repulsive fixed point if $|\lambda|<1$. The idea behind this claim is that, if $|\lambda|<1$, then given a point $w$ in a neighbourhood of $z=\infty$ we have the inequality $\left|O_{n, \alpha}(w)\right| \approx$ $|\lambda||w|<|w|$. Alternatively, we can perform the change of variables $I(z)=1 / z$ which sends $z=\infty$ to $z=0$. It is not difficult to check that after this change of variables the fixed point $z=0$ has multiplier $\left(I \circ O_{n, \alpha} \circ I\right)^{\prime}(0)=1 / \lambda$ and hence it is repelling if and only if $|\lambda|<1$. By using this $\lambda$ the proof the following lemma is straightforward.

Proposition 6.1. The fixed point $z=\infty$ satisfies the following statements.

1. If $\left|\alpha-\frac{1-4 n+5 n^{2}}{2(n-1)(2 n-1)}\right|<\left(\frac{n}{2(2 n-1)}\right)$, then $z=\infty$ is an attractor. In particular, it is a superattracting fixed point if its multiplier is equal to 0, i.e. $\alpha=\frac{n}{n-1}$.

2. If $\left|\alpha-\frac{1-4 n+5 n^{2}}{2(n-1)(2 n-1)}\right|=\left(\frac{n}{2(2 n-1)}\right)$, then $z=\infty$ is an indifferent point.

3. If $\left|\alpha-\frac{1-4 n+5 n^{2}}{2(n-1)(2 n-1)}\right|>\left(\frac{n}{2(2 n-1)}\right)$, then $z=\infty$ is a repulsive fixed point.

Proof. From Eq. (14) we know that $z=\infty$ is an indifferent fixed point if

$$
\left|\frac{(n-1)(1-2 \alpha-2 n+2 \alpha n)}{2 n(-\alpha-n+\alpha n)}\right|=1 .
$$

We take $\alpha=a+i b, a, b \in R$, in this equation. Then, we calculate the square of the module and simplifying the results we obtain

$$
b^{2}+\left(a-\frac{1-4 n+5 n^{2}}{2(n-1)(2 n-1)}\right)^{2}=\frac{n^{2}}{4(2 n-1)^{2}},
$$

that corresponds to the equation of a circle of center $\left(\frac{1-4 n+5 n^{2}}{2(n-1)(2 n-1)}, 0\right)$ and radius $\frac{n}{2(2 n-1)}$.

The previous result is the analogous to Proposition 2.1 for the family $O_{\alpha}(z)$. Indeed, it is easy to check that the circles of parameters given above coincide for $n=2$. The only difference is that the change of variables performed in the family $O_{\alpha}(z)$ sends the two roots of the polynomial $z^{2}+c$ to $z=0$ and $z=\infty$. This change of variables also sends $\infty$ to $z=1$.

The operators $O_{n, \alpha}(z)$ posses a symmetry property which will be interesting for us. It is described in the following lemma. Its proof is analogous to the one of Lemma 3.1 .

Lemma 6.2. Let $n \in \mathbb{N}$ and let $\xi$ be an nth-root of the unity, i.e. $\xi^{n}=1$. Then $I_{\xi}(z)=\xi z$ conjugates $O_{n, \alpha}(z)$ with itself, i.e.

$$
I_{\xi} \circ O_{n, \alpha}(z)=O_{n, \alpha} \circ I_{\xi}(z) .
$$


This lemma tells us that the orbits of points placed in symmetric positions with respect to the $n t h$-roots of the unity are also symmetric. It is useful since, as we shall see, all free critical points are preserved by this symmetry. Critical points are given by the solutions of $O_{n, \alpha}^{\prime}(z)=0$, where $O_{n, \alpha}^{\prime}(z)$ is given by

$$
\frac{\left(z^{n}-1\right)^{2}(n-1)\left(\alpha\left(1-2 n+n^{2}\right)+\alpha^{2}\left(-2+4 n-2 n^{2}\right)+\left(2 n^{2}-n+\alpha\left(-1+5 n-4 n^{2}\right)+\alpha^{2}\left(2-4 n+2 n^{2}\right)\right) z^{n}\right)}{z^{n}\left(2 n\left(n z^{n}-\alpha(n-1)\left(z^{n}-1\right)\right)^{2}\right)} .
$$

Hence, the $n t h$-roots of the unity are double solutions of the equation $O_{n, \alpha}^{\prime}(z)=0$. Indeed, they are superattracting fixed points of local degree 3 of the operator by construction of the numerical method. We also have $n$ other critical points which are solutions of

$\alpha\left(1-2 n+n^{2}\right)+\alpha^{2}\left(-2+4 n-2 n^{2}\right)+\left(2 n^{2}-n+\alpha\left(-1+5 n-4 n^{2}\right)+\alpha^{2}\left(2-4 n+2 n^{2}\right)\right) z^{n}=0$.

We denote them by

$$
c_{n, \alpha, \xi}=c_{\xi}=\xi\left(\frac{\alpha\left(-1+2 n-n^{2}\right)+\alpha^{2}\left(2-4 n+2 n^{2}\right)}{2 n^{2}-n+\alpha\left(-1+5 n-4 n^{2}\right)+\alpha^{2}\left(2-4 n+2 n^{2}\right)}\right)^{1 / n},
$$

where $\xi$ denotes the $n t h$-root of the unity. Since these $n$ free critical points are symmetric with respect to the $n t h$-roots of the unity, it follows from Lemma 6.2 that it is enough to analyse the asymptotic behaviour of one of the $n$ critical orbits to comprehend it for all of them.

In the following result we show that, fixed $n \geq 2$, there exists a unique parameter $\alpha(n)$ such that the numerical method associated to $O_{n, \alpha(n)}$ has order of convergence 4 . This result was also obtained in [?], from the point of view of real dynamical systems.

Proposition 6.3. Fixed $n \geq 2$, the numerical method associated to $O_{n, \alpha}(z)$ has order of convergence 4 if, and only if, $\alpha$ is given by

$$
\alpha(n)=\frac{2 n-1}{3 n-3}
$$

Proof. A parameter $(n, \alpha)$ is associated to a numerical method which has order of convergence 4 if, and only if, the critical points $c_{n, \alpha, \xi}$ coincide with the roots of the unity. Indeed, these are the only parameters for which the roots are critical points of multiplicity 3 and, hence, are superattracting fixed points of local degree 4.

The critical points defined in Equation (15) coincide with the roots of the unity $\xi$ if

$$
\frac{\alpha\left(-1+2 n-n^{2}\right)+\alpha^{2}\left(2-4 n+2 n^{2}\right)}{2 n^{2}-n+\alpha\left(-1+5 n-4 n^{2}\right)+\alpha^{2}\left(2-4 n+2 n^{2}\right)}=1 .
$$

Isolating $\alpha$ in this equation we obtain $\alpha=\frac{2 n-1}{3 n-3}$.

We want to point out that we have not described yet all the critical points of the family. Indeed, it is known that any degree $d$ rational map has $2 d-2$ critical points (c.f. [?] or [?]). The maps $O_{n, \alpha}(z)$ have degree $2 n$, which is the maximum of the exponents of $z$ over the denominator and the numerator of the operator. Hence, it has $4 n-2$ critical points. However, we have only found $3 n$ of them when solving the equation. This is because we solved the equation in $\mathbb{C}$. The equation $O_{n, \alpha}^{\prime}(z)=0$ should be solved over the Riemann sphere $\widehat{\mathbb{C}}$. This means that $z=\infty$ and the poles can also be critical points, but they can not be found by solving the equation 
directly. This can be solved by performing a change of variables near $z=\infty$. However, we do not need to do this change to know if $z=\infty$ is a critical point since we have already studied its local behaviour as fixed point (see Lemma 6.1). On the other hand, the critical points which are preimages of $z=\infty$ are not enough to allow the stability of $z=\infty$. Indeed, the basin of attraction of an attracting fixed point (not superatracting) contains a critical point whose orbit accumulates on the fixed point but it is never mapped onto it (c.f. [?, Lemma 8.5]).

Since all free critical points $c_{\xi}$ are symmetric and, by Lemma 6.2, have symmetric orbits, to find all possible stable dynamics of the maps $O_{n, \alpha}(z)$ other than the superattracting fixed points coming from the roots of the polynomial $z^{n}-1$ and the fixed point $z=\infty$ (see Proposition 6.1) it is enough to analyse the asymptotic behaviour of the orbit of one of the free critical points. Consequently, we can attempt to draw the parameter spaces of the maps $O_{n, \alpha}(z)$ for several fixed $n$. In Figure 7 we show the parameter spaces for $n=2,3,4,5,10$, and 100 . The pictures are done by using a $C$ program similar to the one used for the operator $O_{\alpha}(z)$. In this case we iterate the critical point $c_{n, \alpha, 1}$ up to 150 times, where 1 denotes the choice of the root of the unity. If in a number of iterations smaller than 150 the iteration $w$ is close enough to the root $\xi$ of the polynomial $\left(|\xi-w|<10^{-4}\right)$ then we conclude that the critical orbit converges to a $\xi$ and stop iterating. In that case we plot the parameter $\alpha$ using a scaling from pallid blue (slow convergence) to green to yellow and to red (fast convergence) depending on how fast the critical orbit converges to the root. If after 150 iterates the critical orbit has not reached the neighbourhood of a root of $z^{n}-1$ then we plot the parameter $\alpha$ in black. We want to point out that, for all pictures, we have used the range of $\alpha$ given by $\operatorname{Re}(\alpha) \in(-1.4,4.6)$ and $\operatorname{Im}(\alpha) \in(-2,2)$ and a grid of $3000 \times 2000$ points. We do not show the numerics outside this range since we have found no pair $(n, \alpha)$ for which the the orbit of $c_{n, \alpha, 1}$ does not converge to a zero of $z^{n}-1$ outside it. Consequently, we observe how the bound given in Theorem 4.1 for the boundedness of the Cat set $(n=2)$, whose proof depends strongly on the choice of the polynomials $z^{2}+c$, is also satisfied when we study numerically the parameter space for arbitrary $n$. In all figures we can see the black disk of parameters described in Proposition 6.1 for which $z=\infty$ is an attracting fixed point. It follows from Proposition 6.1 that the size of this disk decreases when $n$ increases and its radius converges to $1 / 4$ when $n \rightarrow \infty$. We also observe that the necklace of the cat set grows thicker with $n$ and that for parameters close to the necklace the critical orbits take more iterates to converge to a root. For $n=10$ we observe some small blacks disks in the necklace. They correspond to parameters for which 150 iterates are not enough for the critical orbits to converge to a root. If we would increase the number of iterates these disks would become much smaller. For $n=100$ these small disks become relatively big black components of a size comparable to the head of the generalized Cat set. Again these black regions of parameters would become smaller increasing the number of iterates. We also want to point out that the generalized Cat set moves slightly to the left when we increase $n$.

We finish the section providing some pictures of the dynamical planes of the operators $O_{n, \alpha}(z)$. The pictures are done using a program in $\mathrm{C}$ which is similar to the one used for the parameter spaces. In this case, we fix $n$ and $\alpha$ and iterate the points in a grid of $1000 \times 1000$ points. As before, if in a number of iterations smaller than 150 the point $w$ is close enough to a root $\xi$ of the polynomial $\left(|\xi-w|<10^{-4}\right)$ then we plot the corresponding point in the grid using a scaling from pallid blue (slow convergence) to green to yellow and to red (fast convergence). If we reach 150 iterates without converging, we plot the point of the grid in black. In Figure 8 we show the dynamical planes of 4 pairs $(n, \alpha=n /(n-1))$ such that $z=\infty$ is a superattracting fixed point (see Proposition 6.1). We observe how the basins of attraction of the roots become smaller when we increase $n$. 


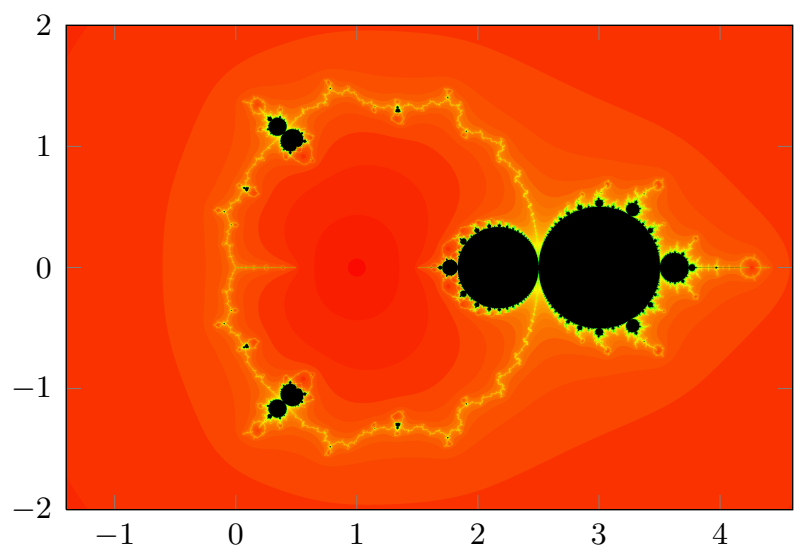

(a) $n=2$

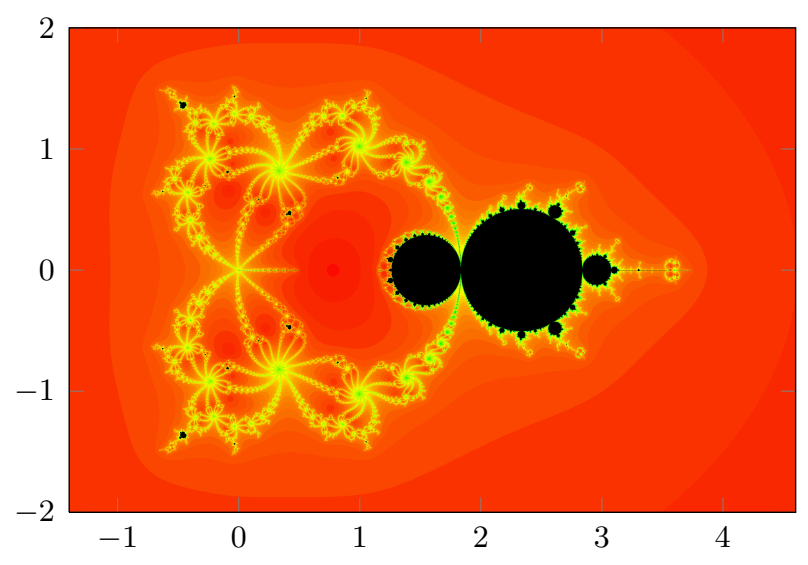

(c) $n=4$

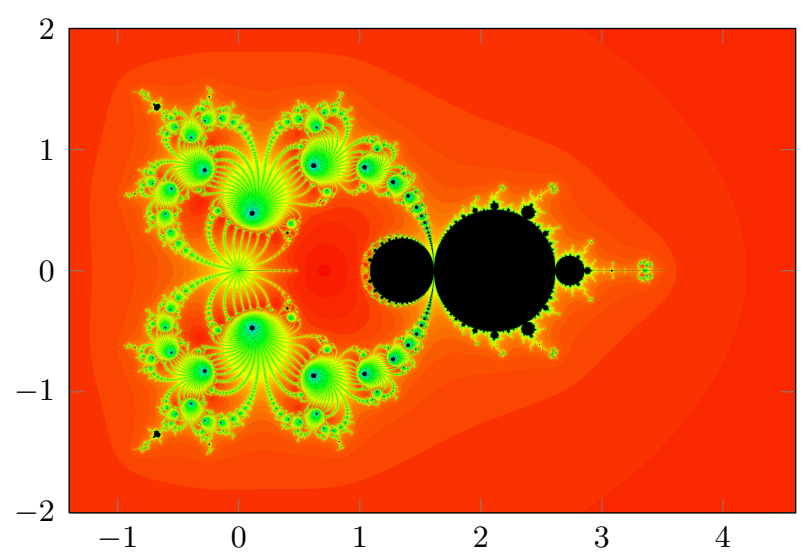

(e) $n=10$

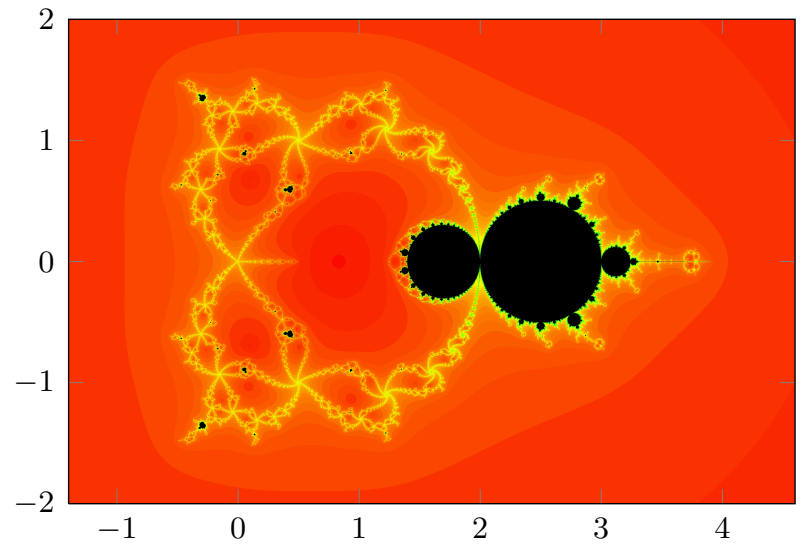

(b) $n=3$

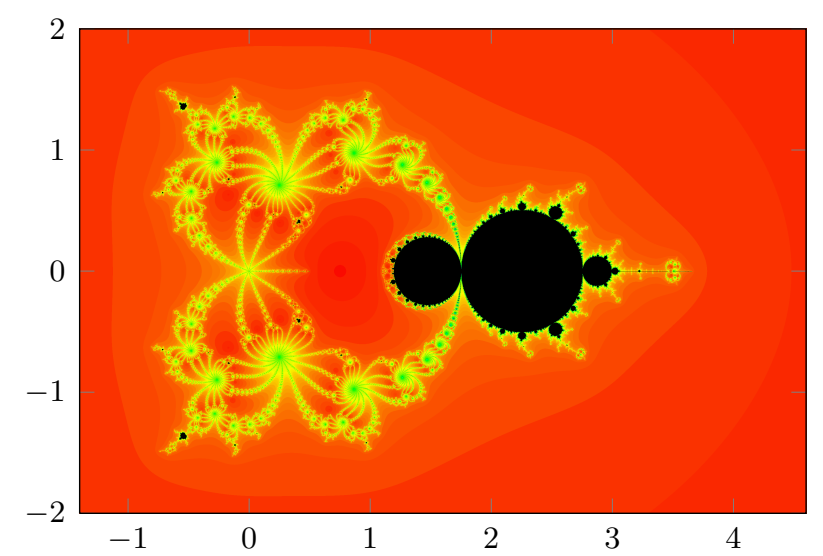

(d) $\mathrm{n}=5$

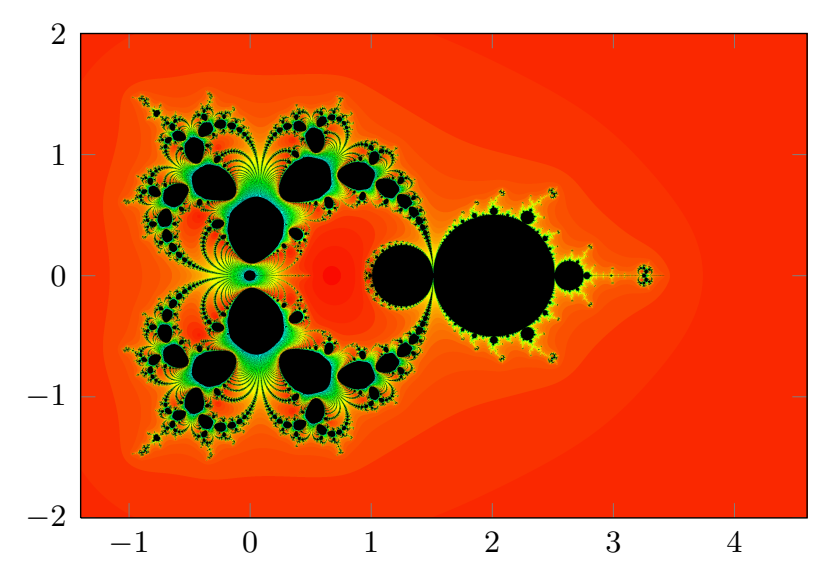

(f) $n=100$

Figure 7: Parameter space of the family $O_{\alpha, n}(z)$ for different values of $n$. For all pictures the range of $\alpha$ is $\operatorname{Re}(\alpha) \in(-1.4,4.6)$ and $\operatorname{Im}(\alpha) \in(-2,2)$. 


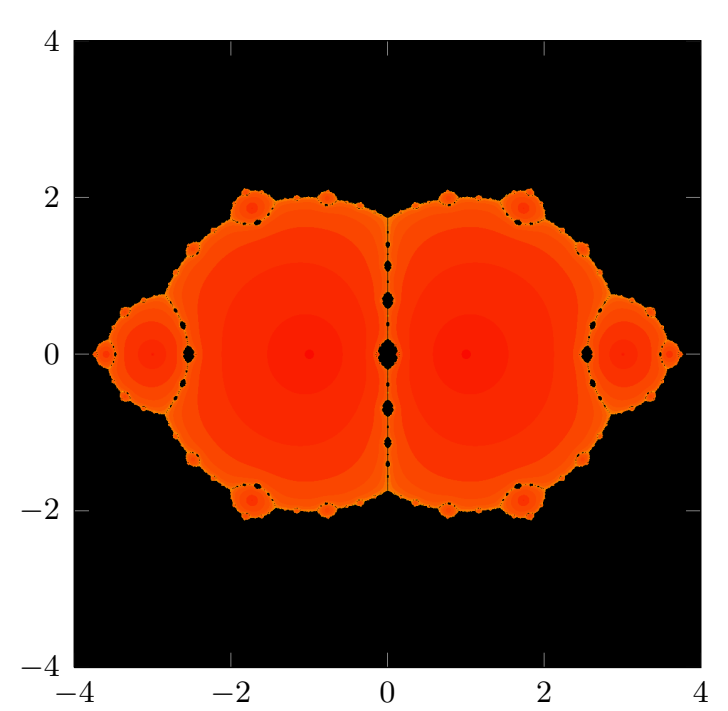

(a) $\mathrm{n}=2$

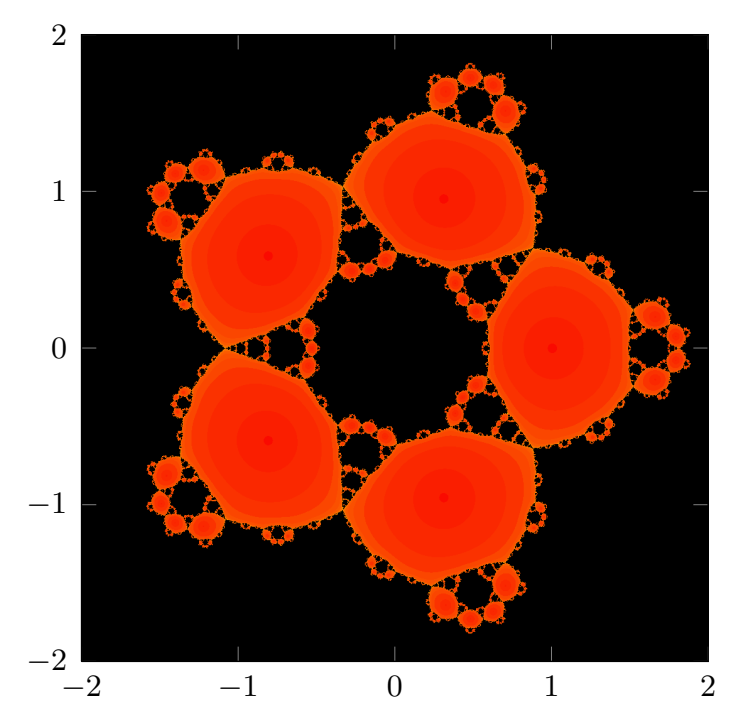

(c) $\mathrm{n}=5$

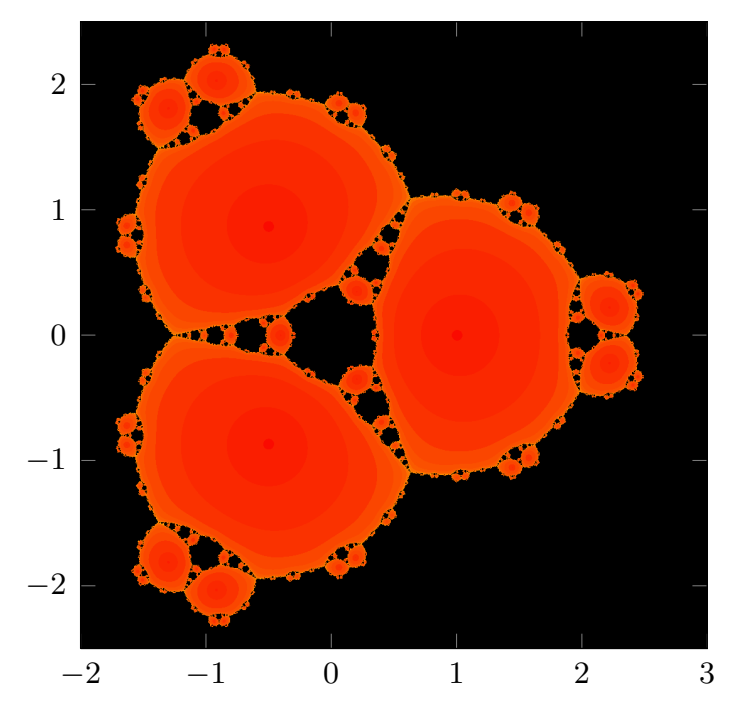

(b) $n=3$

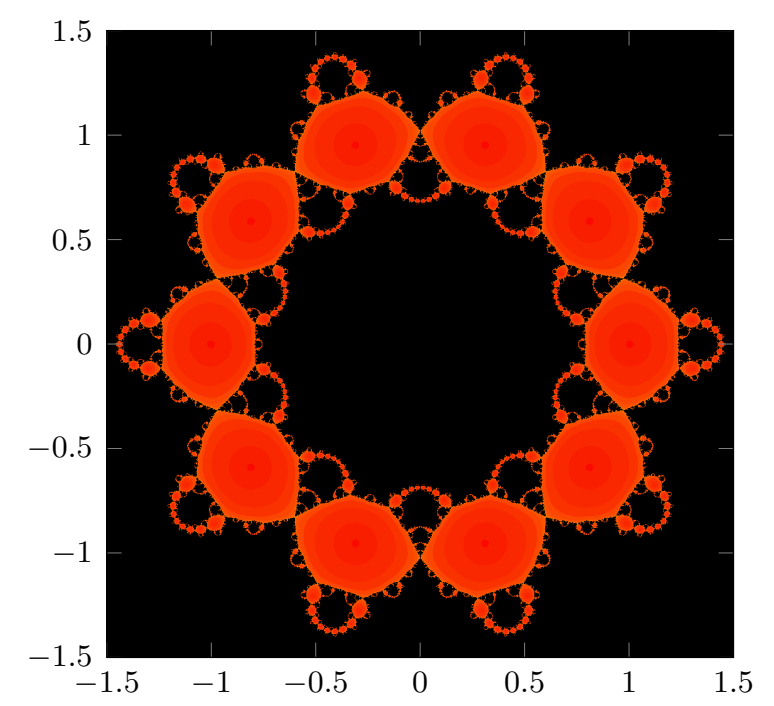

(d) $\mathrm{n}=10$

Figure 8: Dynamical planes of the operators $O_{n, \alpha}(z)$ for $n=2,3,5,10$ and $\alpha=n /(n-1)$.

In Figure 9 we provide 4 pictures of parameters for which there is no stable behaviour other than the basins of attraction of the roots. Figures (a) and (c) correspond to parameters which belong to the red region bounded by the necklace of the parameter spaces. Figures (b) and (d) correspond to parameters on the unbounded red region surrounding the necklace in the parameter space. We observe that the convergence is slower when we increase $n$. We also observe that the convergence is faster in the small red region bounded by the necklace. It is not surprising given that the parameter $\alpha(n)$ for which the method has order of convergence 4 (see Proposition 6.3) lies in this region for all parameter spaces we have plot (see Figure 7). In all pictures we observe small regions of slow convergence. They correspond to regions which surround preimages of $z=\infty$. Among those regions, the bigger one surrounds $z=0$, which is a preimage of order $n-1$ of $z=\infty$ due to the term $z^{n-1}$ in the denominator of $O_{n, \alpha}(z)$ (Equation (13)). 


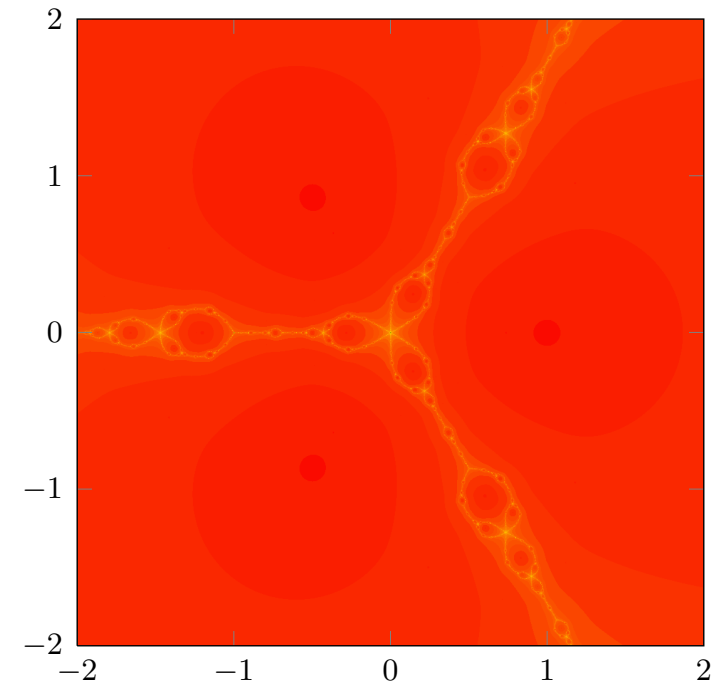

(a) Dynamical plane of $O_{3,0.75}(z)$.

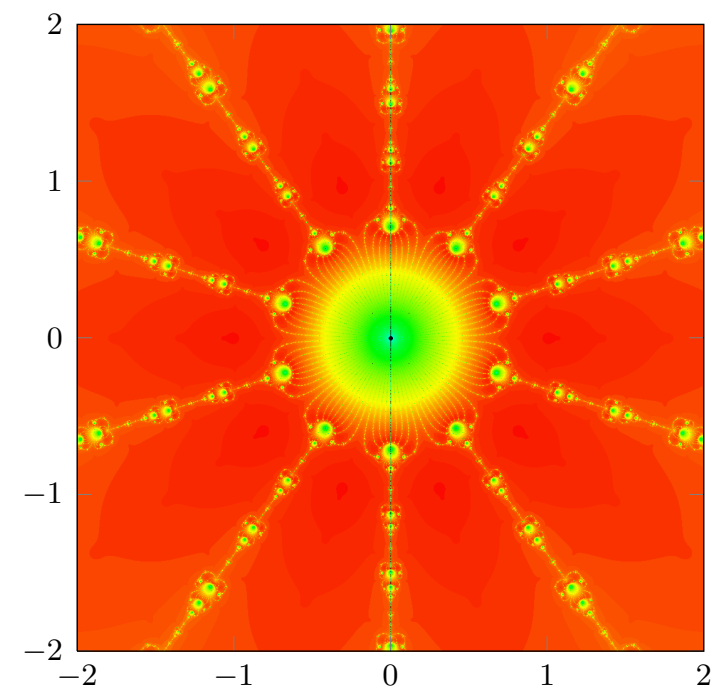

(c) Dynamical plane of $O_{10,0.75}(z)$.

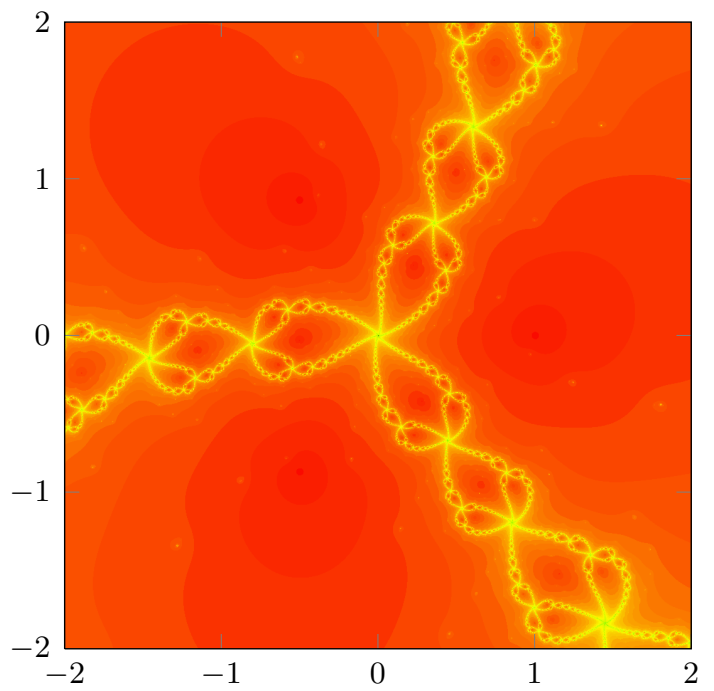

(b) Dynamical plane of $O_{3,4 i}(z)$.

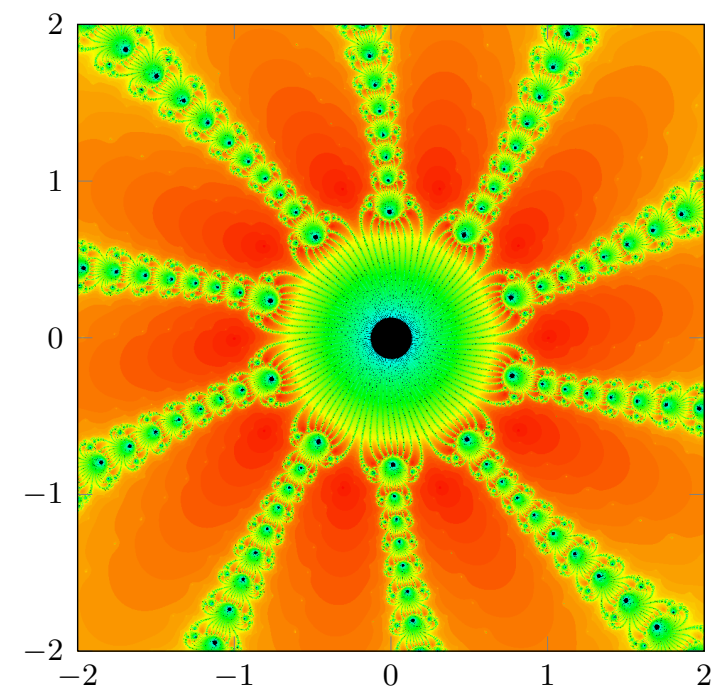

(d) Dynamical plane of $O_{10,4 i}(z)$.

Figure 9: Dynamical planes of 4 operators $O_{n, \alpha}(z)$ for which there is no stable behaviour other than the basins of attraction of the roots of the polynomials.

Finally, in Figure 10 we plot the dynamical plane of $O_{n, \alpha}(z)$ for $n=100$ and $\alpha=1$, which corresponds to the super-Halley's method. We observe that the basins of attraction of the roots (in red) have a rather small size. Notice that the roots lie in the inner red 'annulus' of the left figure, which contains the unit circle. We observe an outer 'annulus' which corresponds to a preimage of the original one. By taking a bigger range of the picture we can observe many more of these 'annular' preimages. We want to remark that for $n$ big some numerical problems appear. Indeed, standard arguments of complex dynamics show that small preimages of the red regions should be observable near $z=0$, since it is a preimage of the fixed point $z=\infty$ which is repelling for $n=100$ and $\alpha=1$ (c.f. Proposition 6.1). 

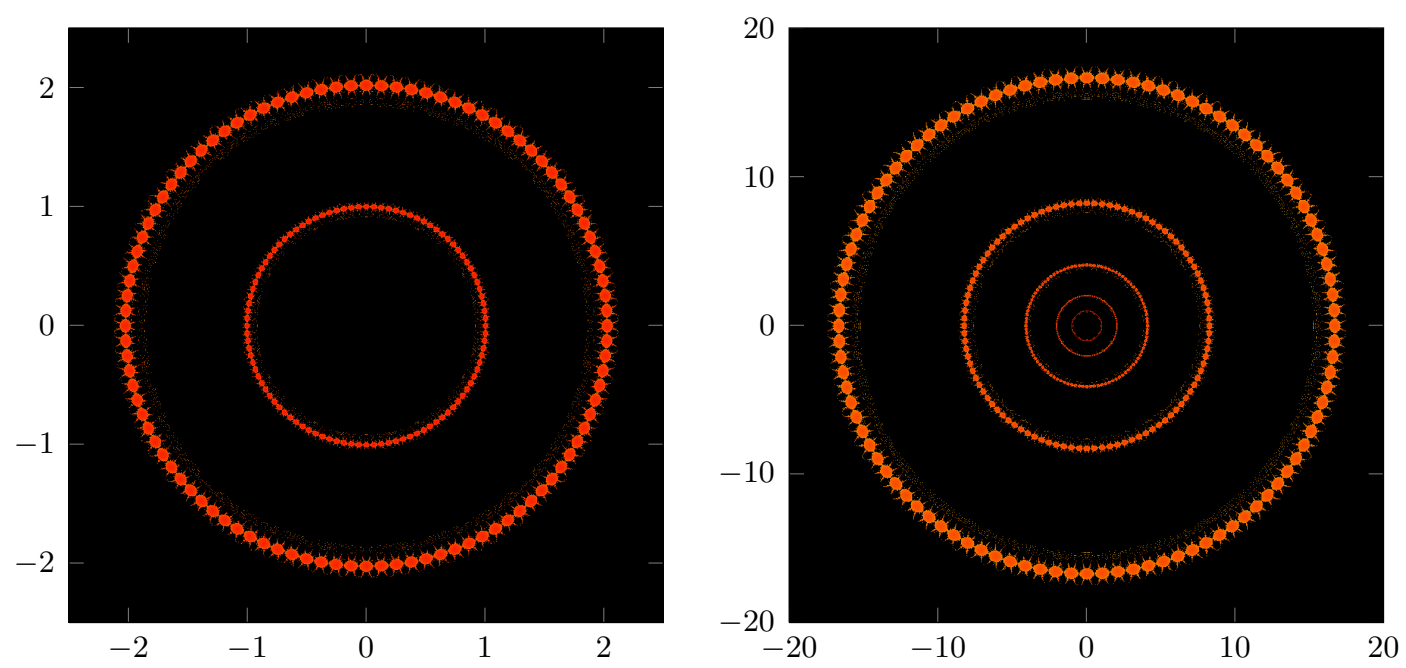

Figure 10: Dynamical plane of $O_{100,1}(z)$.

\section{Conclusions}

In this paper, we study the Chebyshev-Halley methods using the family of degree $n$ polynomials $z^{n}+c$. We find $n$ free critical points and prove that they are symmetric with respect to the $n$ th-roots of the unity. Hence, it is enough to analyze the asymptotic behaviour of one of the $n$ critical orbits to comprehend the asymptotic behaviour for all of them. We also show that infinity is a fixed point, but it is not always attracting (Proposition 6.1). Moreover, we observe that when infinity is attracting, the basins of attraction of the roots become smaller as the value of $n$ grows. Taking into account these results we draw the parameter spaces of this family of maps for $\mathrm{n}=2,3,4,5,10,100$. In all figures, we can see the black disk of parameters described in Proposition 6.1 for which the strange fixed point $z=\infty$ is attracting. Afterwards, we show the dynamical planes for $\mathrm{n}=2,3,5,10$ and $\alpha=\frac{n}{n-1}$. For these parameters $z=\infty$ is superatracting (see Proposition 6.1). We observe that the basins of attraction of the roots become smaller when $n$ increases.

Although the order of convergence of the Chebyshev-Halley family is 3, we demonstrate that there is a member of order 4 for each value of $n$ (Proposition 6.3). Fixed $n$, this parameter belongs to a hyperbolic component of parameters bounded by the necklace (see Figure 7). The convergence to the roots for parameters within this region seems faster than in the unbounded hyperbolic component surrounding the necklace (see Figure 9p. However, as we increase $n$, the generalized Cat set moves slightly to the left an this hyperbolic region of parameters with faster convergence becomes smaller (see Figure 7). Indeed, we can observe that the parameter $\alpha=1$, which corresponds to the super-Halley's method and has order of convergence 4 for $n=2$ (c.f. Proposition 6.3), has good behaviour for $n$ small and bad behaviour for $n=100$ (see Figure 10).

We also continue the dynamical study of the Chebyshev-Halley family applied on quadratic polynomials. For this case, we bound the set of parameters corresponding to iterative methods with no stable behaviour (Proposition 4). That means that if $\alpha>8.57$, the orbit of almost any initial condition converges to one of the roots. We can also observe that the bound on the set of parameters with bad numerical behaviour obtained in the case of the quadratic polynomials $z^{2}+c$ also applies for the $z^{n}+c$. 
Acknowledgments: The authors would like to thank the anonymous referees for their suggestions and comments, which improved this paper.

Acknowledgments: The first and third authors were supported by the Spanish project MTM2014-52016-C02-2-P, the Generalitat Valenciana Project PROMETEO/2016/089 and UJI project P1.1B20115-16. The second author was supported by RedIUM and MINECO (Spain) through the research network MTM2014-55580-REDT and by the mathematics institute IMAC (Castellón, Spain).

\section{References}

[1] S. Amat, S. Busquier, and S. Plaza. Review of some iterative root-finding methods from a dynamical point of view. Sci. Ser. A Math. Sci., 10:3-35, 2004.

[2] S. Amat and S. Busquier. Ed. Advances in iterative Methods for Nonlinear Equations, volume 10 of SEMA SIMAI. Springer International Publishing Switzerland, Switzerland, 2016. Iterative methods.

[3] I.K. Argyros and Á. A. Magreñán. On the local convergence and the dynamics of Chebyshev-Halley methods with six and eight order of convergence. J. of Comput. App. Math., 298:236251, 2016.

[4] I.K. Argyros and Á. A. Magreñán. A study on the local convergence and the dynamics of Chebyshev-Halley type methods free from second derivative. Numer. Algor., 71:1-23, 2016.

[5] A. F. Beardon. Iteration of rational functions, volume 132 of Graduate Texts in Mathematics. Springer-Verlag, New York, 1991. Complex analytic dynamical systems.

[6] P. Blanchard. The dynamics of Newton's method, volume 49 of Proc. Sympos. Appl. Math. Amer. Math. Soc., Providence, RI, 1994. Complex dynamical systems (Cincinnati, OH, 1994).

[7] A. D. Bryuno. On convergence of transforms of differential equations to the normal form. Dokl. Akad. Nauk SSSR, 165:987-989, 1965.

[8] B. Campos, A. Cordero, Á. A. Magreñán, J. R. Torregrosa, and P. Vindel. Study of a biparametric family of iterative methods. Abstr. Appl. Anal., page 12, 2014.

[9] B. Campos, A. Cordero, J. R. Torregrosa, and P. Vindel. Dynamics of the family of c-iterative methods. Int. J. Comput. Math., 92(9):1815-1825, 2015.

[10] J. Canela, N. Fagella, and A. Garijo. Tongues in degree 4 Blaschke products. Nonlinearity, 29(11):34643495, 2016.

[11] A. Cordero, J. García-Maimó, J. R. Torregrosa, M. P. Vassileva, and P. Vindel. Chaos in King's iterative family. Appl. Math. Lett., 26(8):842-848, 2013.

[12] A. Cordero, J. R. Torregrosa, and P. Vindel. Bulbs of period two in the family of Chebyshev-Halley iterative methods on quadratic polynomials. Abstr. Appl. Anal., page 10, 2013. 
[13] A. Cordero, J. R. Torregrosa, and P. Vindel. Dynamics of a family of Chebyshev-Halley type methods. Appl. Math. Comput., 219(16):8568-8583, 2013.

[14] A. Cordero, J. R. Torregrosa, and P. Vindel. Period-doubling bifurcations in the family of Chebyshev-Halley type methods. International Journal of Computer Mathematics, 90(10):2061-2071, 2013.

[15] M. A. Hernández J. M. Gutiérrez and N. Romero. Dynamics of a new family of iterative processes for quadratic polynomials. Journal of Computational and Applied Mathematics, 233:2688-2695, 2010.

[16] M. A. Hernández J. M. Gutiérrez and M.A. Salanova. Calculus os $n$th roots and third order iterative methods. Nonlinear Analysis, 47(4):2875-2880, 2001.

[17] K. Kneisl. Julia sets for the super-Newton method, Cauchy's method, and Halley's method. Chaos, 11(2):359-370, 2001.

[18] J. Milnor. Dynamics in one complex variable, volume 160 of Annals of Mathematics Studies. Princeton University Press, Princeton, NJ, third edition, 2006.

[19] C. L. Siegel. Iteration of analytic functions. Ann. of Math. (2), 43:607-612, 1942.

[20] D. Sullivan. Quasiconformal homeomorphisms and dynamics I. Solution of the Fatou-Julia problem on wandering domains. Ann. of Math. (2), 122(3):401-418, 1985. 